\title{
Variational water-wave model with accurate dispersion and vertical vorticity
}

\author{
Colin Cotter • Onno Bokhove
}

Received: 28 February 2009 / Accepted: 30 September 2009 / Published online: 28 October 2009

(C) The Author(s) 2009. This article is published with open access at Springerlink.com

\begin{abstract}
A new water-wave model has been derived which is based on variational techniques and combines a depth-averaged vertical (component of) vorticity with depth-dependent potential flow. The model facilitates the further restriction of the vertical profile of the velocity potential to $n$-th order polynomials or a finite-element profile with a small number of elements (say), leading to a framework for efficient modelling of the interaction of steepening and breaking waves near the shore with a large-scale horizontal flow. The equations are derived from a constrained variational formulation which leads to conservation laws for energy, mass, momentum and vertical vorticity. It is shown that the potential-flow water-wave equations and the shallow-water equations are recovered in the relevant limits. Approximate shock relations are provided, which can be used in numerical schemes to model breaking waves.
\end{abstract}

Keywords Bores · Coastal engineering · Variational principles · Wave-current interactions

\section{Introduction}

It is always fascinating to watch waves near the shore line. They approach the shore, steepen as the water becomes more shallow, break upon further approach, and run up and down the beach or dike. In Fig. 1, wave trains are seen to approach the shore. Far from the shore, we see that no wave breaking occurs. Upon reaching the shore, part of the wave starts breaking while part of the same wave has steepened significantly without breaking. Finally, waves very near to the shore will break along their entire crest, and subsequently enter the swash zone where the waves run up and down the beach or (rocky) shore.

In hydrodynamics and coastal engineering, depth-averaged shallow-water modelling is, and has been, very successful (e.g. [1]). Due to the depth-averaging, the full three-dimensional fluid-dynamical (multiphase, Navier-Stokes or Euler) equations of motions are reduced in complexity. Only dependence on horizontal coordinates and time

C. Cotter

Department of Aeronautics, Imperial College, London, UK

O. Bokhove $(\varangle)$

Department of Mathematics, Institute of Mechanics, Processes and Control, Twente, University of Twente, P.O. Box 217, 7500 AE Enschede, The Netherlands

e-mail: o.bokhove@math.utwente.nl 

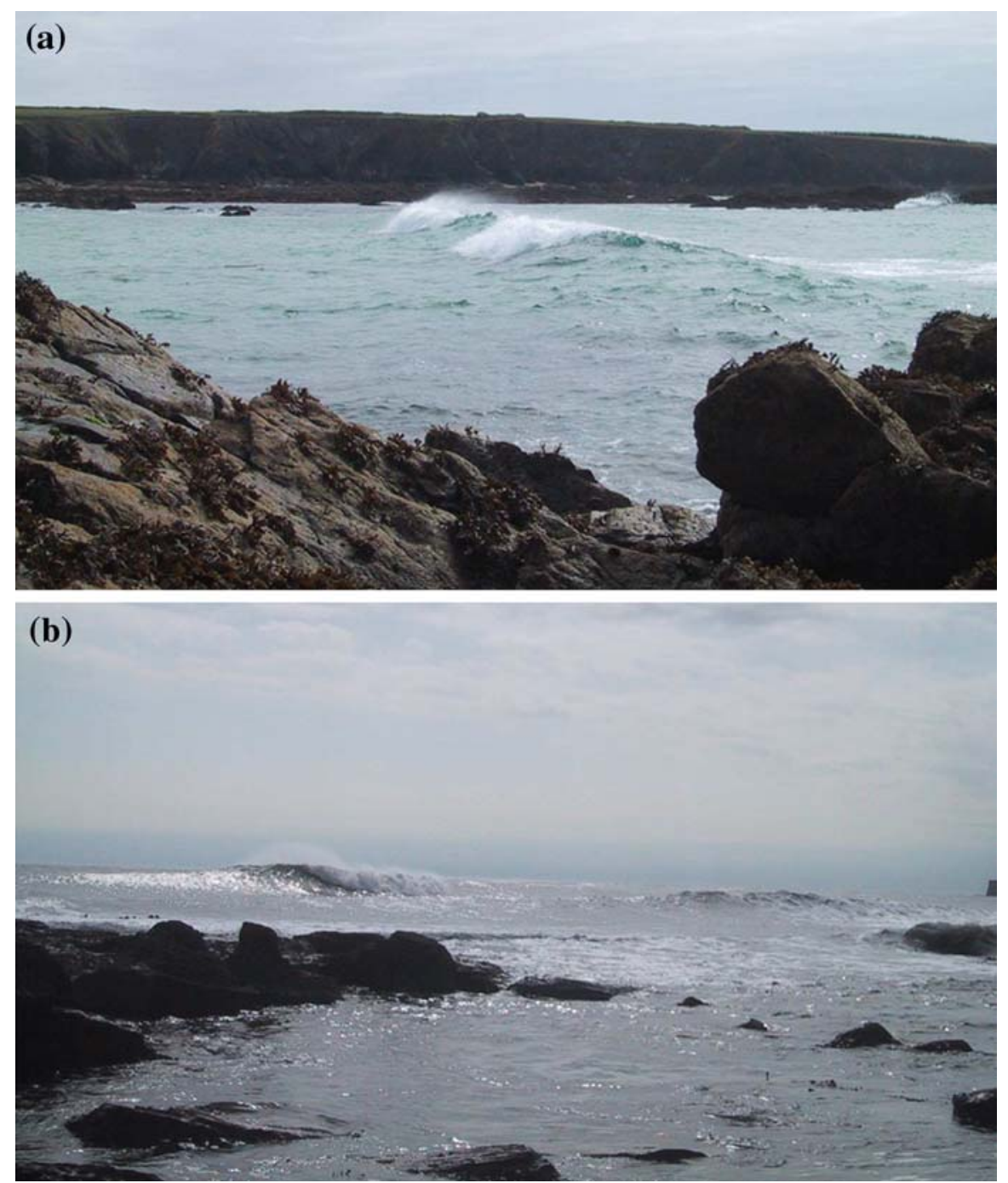

Fig. 1 a A steepening wave with partly broken and unbroken crest is approaching the shoreline. b Two different waves with broken and steepening crests approach the shore line. Photograph: D. Howell Peregrine

is then retained. The vertical coordinate $z$ is aligned opposite to the acceleration of gravity $g$, and wave breaking implies that the free surface becomes multivalued. The free surface lies at $z=h(x, y, t)+b(x, y)$ relative to $z=0$, with water depth $h=h(x, y, t)$ and fixed bottom topography $b=b(x, y)$ a function of the horizontal coordinates $x$ and $y$, and time $t$. A defining sketch is given in Fig. 2. Wave breaking within such a shallow-water model is commonly approximated by using line discontinuities in the horizontal, leading to the well-known bores and hydraulic jumps (see [2, Chap. 10]).

Breaking waves emerge in various forms [3]: plunging breakers are multivalued when viewed along the direction of gravity before the free surface at $z=h+b$ breaks apart, while spilling breakers remain single-valued before the free surface breaks apart. When the free surface breaks apart into spray and foam during wave breaking, the topology of the domain changes, many times. Bores or hydraulic jumps in depth-averaged shallow-water modelling are a straightforward approximation of these breaking waves as localized discontinuities, and when viewed from above these discontinuities form horizontal lines (of finite length). Mass and momentum are conserved across a bore or jump, while kinetic and potential energy are not. Mathematically speaking, these bores and jumps are 

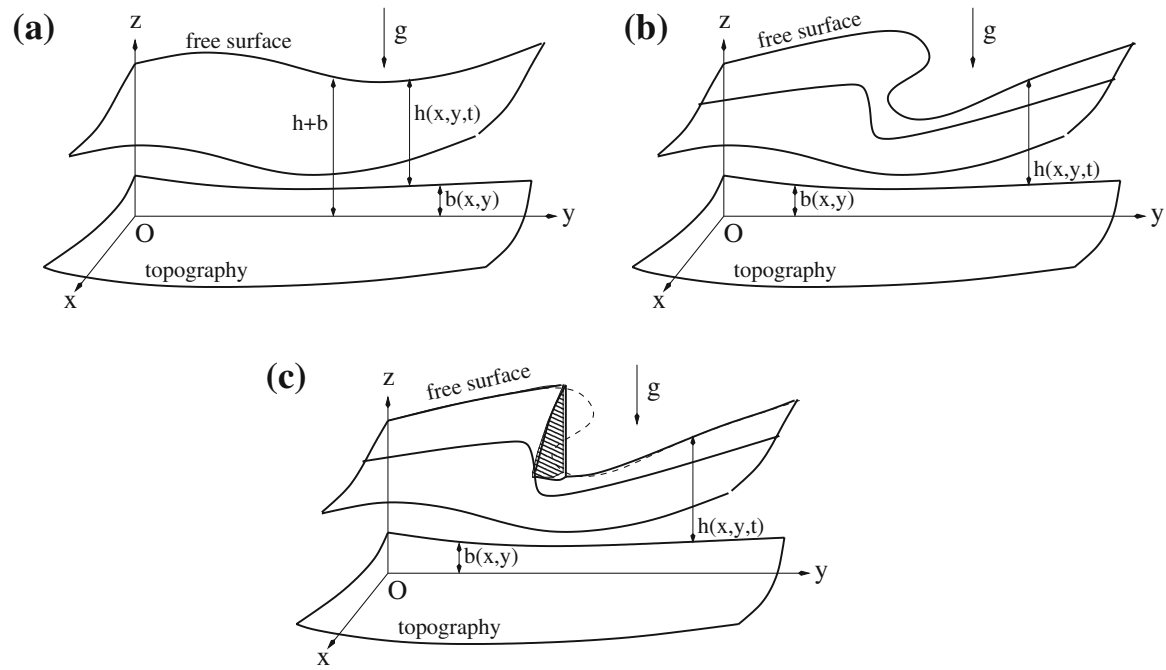

Fig. 2 a A sketch is given to define the coordinates $x, y$ and $z$ as well as the free water surface, the acceleration of gravity $g$, depth $h=h(x, y, t)$ and bottom topography $b=b(x, y)$. b An example of a triple-valued function of the depth $h(x, y, t)$, and $\mathbf{c}$ its bore approximation wherein a line discontinuity is seen, of finite horizontal extent; the vertical face of the bore is patterned; and, the overturning wave approximated is still outlined with a dashed line

akin to shocks in gas dynamics where mass, momentum and total energy conservation are combined with entropy increase. In contrast, energy is lost in shallow-water bores. After all, the dependence on internal energy and entropy in compressible fluids is lost due to the assumed incompressibility of water. While the mathematical theory for gas shocks and hydraulic jumps is the same, the physics in both cases is different.

The simplicity of such a shallow-water model is also its pitfall. Internal wave dispersion is lost as vertical velocity profiles across the depth have been ignored. Boussinesq models are therefore useful in the coastal engineering community, because they include internal wave dispersion to a higher degree of accuracy $[4,5]$. Generally, Boussinesq models are derived from the three-dimensional potential-flow water-wave equations, which describe internal water wave dispersion fully (in the absence of vorticity). Disadvantages of (most) Boussinesq models are threefold. First, dispersion always seems to beat nonlinearity such that wave overturning as the precursor or first stage of wave breaking is always prevented. This is unphysical: waves are observed to break in nature and also do overturn within the potential-flow water-wave model. Second, Boussinesq models tend to be derived from the "parent" water-wave model under the potential-flow assumption, in which case the vertical (component of) vorticity is eliminated from the outset. This contrasts with the derivation of the classical depth-averaged shallow-water model, which contains vertical vorticity consistently from its derivation of the (hydrostatic) incompressible Euler equations. Moreover, in depth-averaged shallow-water models wave breaking along non-uniform bores leads to the generation of potential vorticity anomaly [6-12]. Sometimes, vertical vorticity is added a posteriori to Boussinesq models derived from parent potential-flow water-wave equations. It is unclear whether this is formally justified. Third, Boussinesq models do not always conserve the geometric structure of the parent equations. Hence, preservation of the original variational and Hamiltonian structure with its associated conservation of mass, momentum, and energy is then lost. The variational Boussinesq model of [13] is a notable exception, but is based on the potential-flow ansatz. Other notable exceptions are the Green-Naghdi(-type) models in which the dependence on the vertical coordinate can be approximated in variational principles, within the Lagrangian $[14,15]$ or Eulerian [16] framework; vertical vorticity and the enslaved horizontal components of the vorticity are then included in these latter cases, but the wave dispersion is in principle of lower order than in the model of [13]. As a consequence, wave dispersion appears to be stronger than nonlinearity and wave overturning is never achieved. 
Finally, fully three-dimensional numerical models are in use but computational power is too limited for them to be wave-resolving over large areas. Wave forecasting is therefore generally based on statistical wave modelling. Near the shore for tens of kilometers along the coast and 1-2 km off the coast, deterministic wave and current modelling is required to forecast longshore currents and wave run-up and run-down for flood forecasting. Regions of such size appear feasible for numerical Boussinesq models and shallow-water models, but not for fully three-dimensional ones.

Consequently, our goal is to overcome these various shortcomings. Our aims are to derive a simplified water-wave model with accurate dispersion, nonzero vertical (component of) vorticity, and a bore model for wave breaking. Our derivation will be based on variational techniques: an immediate consequence is that the geometric structure of the parent incompressible Euler equations will be preserved. Conservation of mass, momentum and energy is therefore guaranteed. To illustrate our technique, we will show in passing that Luke's and Miles' variational principles [17, 18] for potential-flow water-wave equations follow by a constraint approximation of the parent variational principle for the incompressible Euler equations. Our model with water-wave dispersion and vertical vorticity is able to describe the wave steepening and breaking, as seen in Figs. 1 and 3, in an advanced treatment of the bore approximation. The broken wave will be modelled as a discontinuity or shallow-water bore as sketched in Fig. 3a. Our model could then also be applied to the propagation of undular bores on rivers, in which dispersion and nonlinearity are balanced in the deeper mid channel, while wave-breaking occurs in shoaling water near the shore lines, along the same wave crest. This steepening and wave-breaking behavior is clearly seen on the Mascaret bore in Fig. 3b, and the Severn bore in Fig. 4.

The outline of our paper is as follows. In Sect. 2, our starting point, the variational principle of the parent incompressible Euler equations, is investigated. To illustrate our manipulations, Luke's and Miles' variational principles are rederived in a perhaps novel way from this starting principle in Sect. 3. Our new model is derived and analyzed in Sect. 4. We conclude with concluding remarks in Sect. 5.

\section{Parent equations-three-dimensional incompressible flow}

\subsection{Variational principle}

A Lagrangian variational principle for the three-dimensional Euler equations with a free surface is summarized next. It forms the foundation for our subsequent approximations of this "parent" variational principle. We start as usual with the construction of a Lagrangian density, $L$, by taking the difference between the kinetic and potential energy density

$L=\frac{1}{2} D|\boldsymbol{u}|^{2}-g D z$

in which $g$ is the acceleration due to gravity, $D=D(x, y, z, t)$ is a (scaled) density, $\boldsymbol{u}=\boldsymbol{u}(x, y, z, t)=(u, v, w)^{T}$ is the fluid velocity vector with horizontal coordinates $(x, y)$ and vertical coordinate $z$, and $t$ is time (see Fig. 2). The density $D$ is the Jacobian between Eulerian coordinates $(x, y, z)$ and Lagrangian label coordinates $\boldsymbol{l}=\left(l_{1}, l_{2}, l_{3}\right)^{T} \in$ $\mathbb{R}^{3}$ for the fluid parcel, such that $D \mathrm{~d} x \mathrm{~d} y \mathrm{~d} z=\mathrm{d} l_{1} \mathrm{~d} l_{2} \mathrm{~d} l_{3}$. The fluid resides in a domain $\Omega$ with solid domain walls $\partial \Omega_{w}$ and a free surface at $\partial \Omega_{s}$, such that $\partial \Omega=\partial \Omega_{w} \cup \partial \Omega_{s}$. The solid walls consist of bottom topography at $z=b(x, y)$ and possibly vertical walls. The free surface has position $z=b(x, y)+h(x, y, t)$; we restrict attention to this single-valued free surface.

Define the flow map $\chi$ such that $\boldsymbol{x}=\chi(\boldsymbol{l}, t)$. Hence, the time derivative $\partial_{t} \equiv \partial / \partial t$ and variation of $\chi$, i.e.,

$\partial_{t} \chi=\boldsymbol{u} \circ \chi \equiv \boldsymbol{u}(\chi, t)$ and $\delta \chi=\mathbf{w} \circ \chi \equiv \mathbf{w}(\chi, t)$,

are the Eulerian velocity and displacement variation mapped back into the Lagrangian label framework. A commonly used initial condition is $\chi(\boldsymbol{l}, 0)=\boldsymbol{l}$ such that the labels $\boldsymbol{l}$ and fluid parcel positions $\boldsymbol{x}=\chi(\boldsymbol{l}, t)$ coincide initially, i.e., at time $t=0$. 

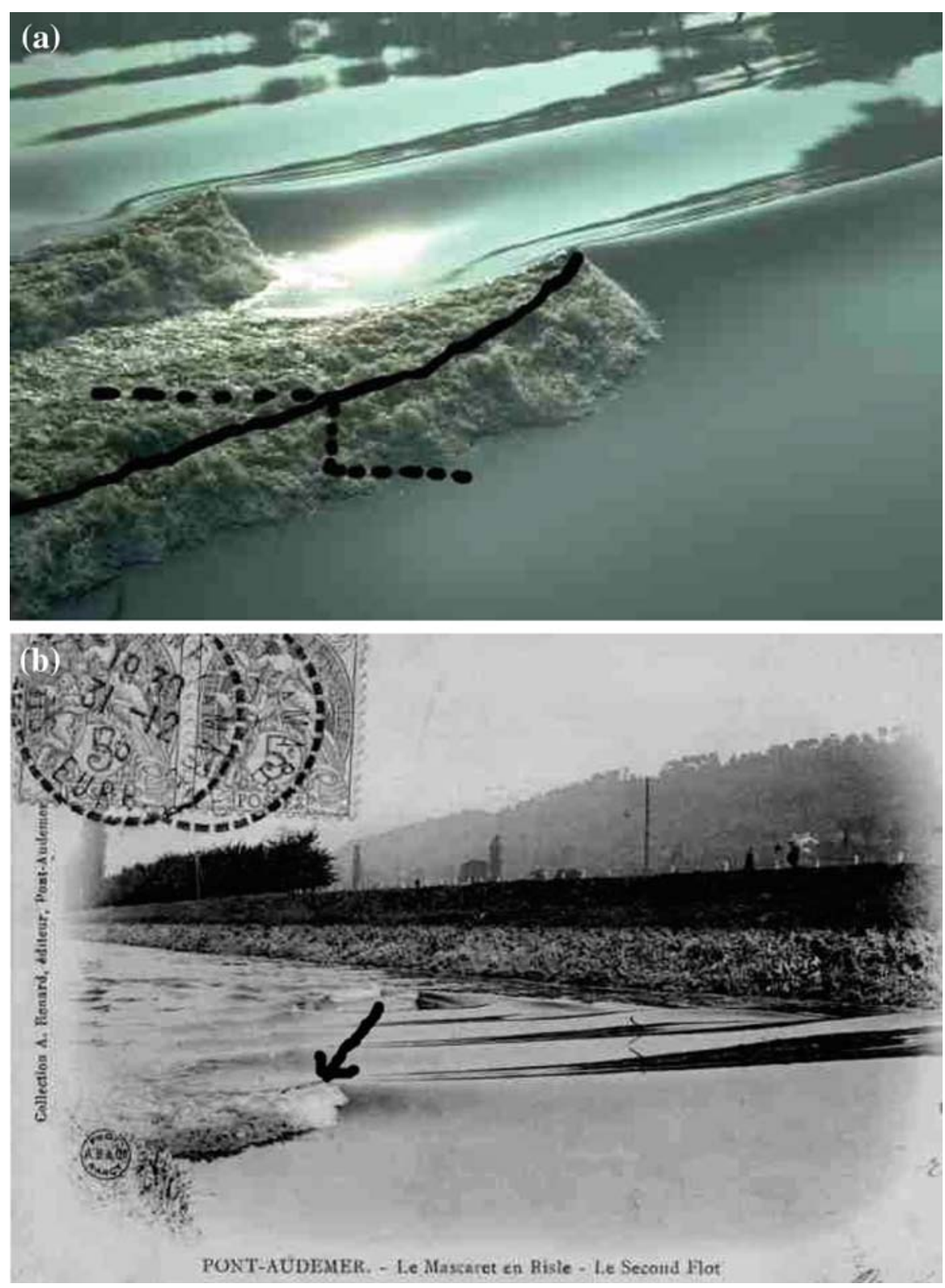

Fig. 3 a In the undular bore on the Severn River the competition between dispersion and nonlinearity is clearly seen. Part of the wave is broken, in the shallower water near the shore, on the left in the picture, and part is not. Photograph: D. Howell Peregrine. The black line added indicates where the bore discontinuity would be, and the dashed line a cross-section of the free-surface approximation of such a bore. b The Mascaret on the Risle, a small tributary of the Seine River at Pont-Audemer, shows the same breaking and non-breaking features on one wave crest. Photograph: J. J. Malandain [19]. The arrow indicates where wave breaking starts. Arrows and lines are added by the authors

We introduce a Lagrange multiplier $p=p(x, y, z, t)$ to enforce the constraint $D-1=0$.

The constrained variational principle then has Lagrangian density $L+p(1-D)$, and reads

$0=\delta \int_{0}^{T} \mathcal{L}[\chi, \dot{\chi}] \mathrm{d} t=\delta \int_{0}^{T} \int_{\Omega} \frac{1}{2} D|\boldsymbol{u}|^{2}-g D z+p(1-D) \mathrm{d} x \mathrm{~d} y \mathrm{~d} z \mathrm{~d} t$, 


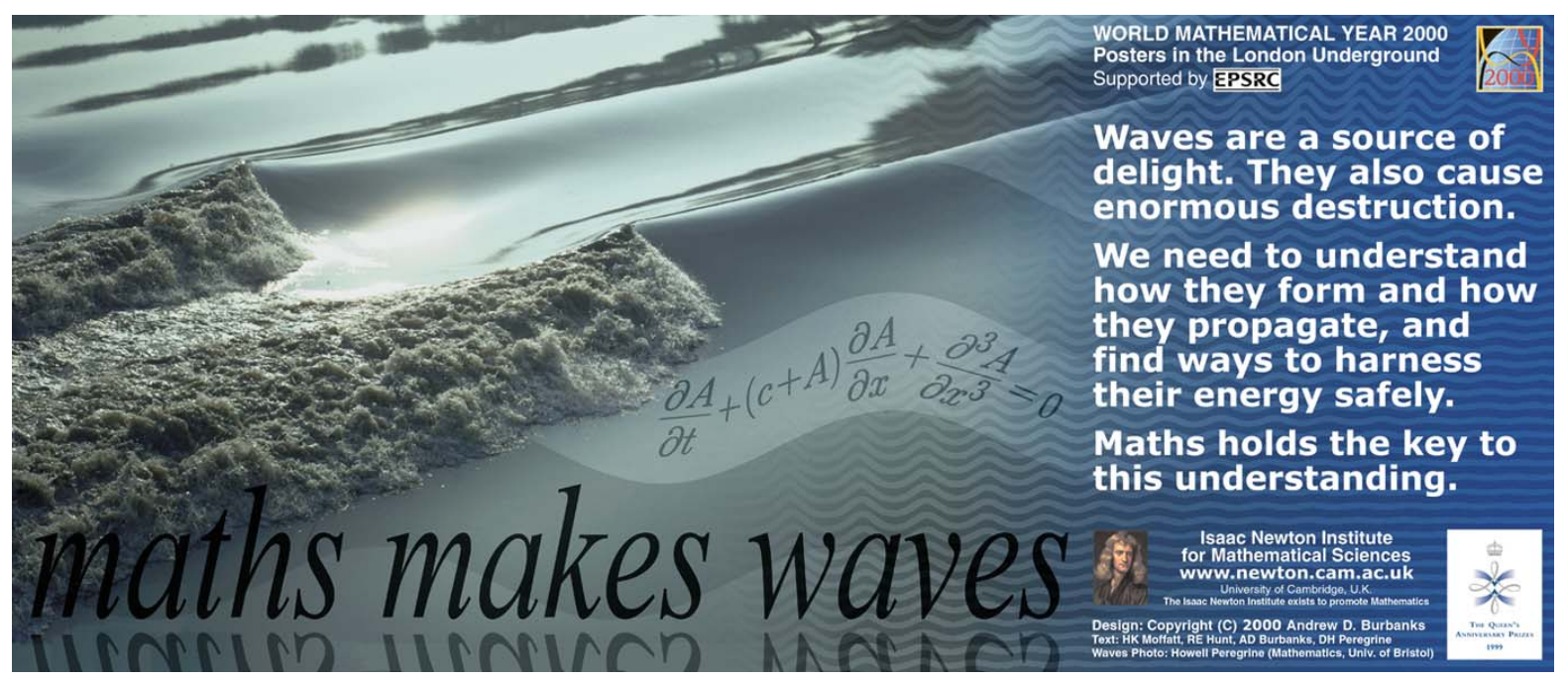

Fig. 4 This "maths makes waves" poster features an undular bore on the Severn River; the competition between dispersion and nonlinearity is clearly seen. Part of the wave is broken on the left, in the shallower water near the shore, and part is not. Photograph: D. Howell Peregrine. Poster design copyright: Andrew Burbanks

spanning a time interval from initial time $t=0$ to $t=T$, in which variations in $D$ and $\boldsymbol{u}$ are related to the Eulerian variation of the label displacements $\mathbf{w}(x, y, z, t)$; see e.g., [20]. Manipulation of (2) and use of the chain rule yields the variation of the velocity to be

$\delta \boldsymbol{u}=\partial_{t} \mathbf{w}+(\boldsymbol{u} \cdot \nabla) \mathbf{w}-(\mathbf{w} \cdot \nabla) \boldsymbol{u}$.

Density $D$ obeys the conservation law

$\partial_{t} D+\nabla \cdot(\boldsymbol{u} D)=0$,

and by analogy its variation relates to the displacement flow $\mathbf{w}$ as follows

$\delta D+\nabla \cdot(\mathbf{w} D)=0$.

The kinematic condition at the free surface $F(x, y, z, t)=z-(h(x, y, t)+b(x, y))=0$ is

$\partial_{t} h+\left(\boldsymbol{v}_{s} \cdot \nabla\right)(h+b)-w_{s}=0$

with the suffix indicating evaluation at the free surface, e.g., $\boldsymbol{u}_{s}=\boldsymbol{u}(x, y, z=h+b, t)=\left(\boldsymbol{v}_{s}, w_{s}\right)$; and, $\boldsymbol{v}=$ $(u, v, 0)^{T}$. Likewise we find the variation

$\delta h+\left(\mathbf{w}_{s} \cdot \nabla\right)(h+b)-\mathbf{w}_{s}=0$

with $\mathbf{w}_{s}=\left(\mathrm{w}_{s 1}, \mathrm{w}_{s 2}, 0\right)^{T}$ the horizontal components of $\mathbf{w}$ evaluated at the surface, and $\mathrm{w}_{s}=\mathrm{w}_{3}$ the vertical component of $\mathbf{w}$ evaluated at the free surface. It is possible to rework (8) and (9) using a horizontal depth-averaged velocity $\overline{\boldsymbol{u}}=\overline{\boldsymbol{u}}(x, y, t)=\int_{b}^{b+h}(u, v)^{T} \mathrm{~d} z / h$ and corresponding label variation $\overline{\mathbf{w}}=\overline{\mathbf{w}}(x, y, t)=\left(\overline{\mathrm{w}}_{1}, \overline{\mathbf{w}}_{2}\right)^{T}$. The results are

$\partial_{t} h+\nabla \cdot(h \overline{\boldsymbol{u}})=0 \quad$ and $\delta h+\nabla \cdot(h \overline{\mathbf{w}})=0$,

in which the gradient is effectively horizontal. The above expressions (5)-(10) are derived in Appendix A. 
Relations (5), (7) and (9) are used to evaluate variations in (4), such that we obtain a Lagrangian density $\mathcal{L}$ as functional of $\boldsymbol{l}, \partial_{t} \boldsymbol{l}$, and $p$; or, $\mathbf{w}$ and $p$ :

$$
\begin{aligned}
0= & \delta \int_{0}^{T} \mathcal{L}\left[\boldsymbol{l}, \partial_{t} \boldsymbol{l}, p\right] \mathrm{d} t \\
= & \delta \int_{0}^{T} \int_{\Omega} \frac{1}{2} D|\boldsymbol{u}|^{2}-g D z+p(1-D) \mathrm{d} x \mathrm{~d} y \mathrm{~d} z \mathrm{~d} t \\
= & \int_{0}^{T} \int_{\Omega} D \boldsymbol{u} \cdot \delta \boldsymbol{u}+\left(\frac{1}{2}|\boldsymbol{u}|^{2}-g z-p\right) \delta D+(1-D) \delta p \mathrm{~d} x \mathrm{~d} y \mathrm{~d} z \mathrm{~d} t+\int_{0} \int_{\partial \Omega_{s}} D_{s} B_{-s} \delta h \mathrm{~d} x \mathrm{~d} y \mathrm{~d} t \\
= & \left.\int_{\Omega}{ }_{D} \boldsymbol{u} \cdot \mathbf{w} \mathrm{d} x \mathrm{~d} y \mathrm{~d} z\right|_{0} ^{T} \\
& -\int_{0}^{T} \int_{\Omega} \mathbf{w} \cdot \partial_{t}(D \boldsymbol{u})+D \boldsymbol{u}(\mathbf{w} \cdot \nabla) \boldsymbol{u}+(D-1) \delta p+\mathbf{w}^{T} \cdot(D u \boldsymbol{u})-D \mathbf{w} \cdot \nabla B_{-} \mathrm{d} x \mathrm{~d} y \mathrm{~d} z \mathrm{~d} t \\
& +\int_{0}^{T} \int_{\partial \Omega_{s}} D_{s}\left(\hat{\mathbf{n}}_{s} \cdot \boldsymbol{u}_{s} \boldsymbol{u}_{s} \cdot \mathbf{w}_{s}-\hat{\mathbf{n}}_{s} \cdot \mathbf{w}_{s} B_{-s}\right) \mathrm{d} s \mathrm{~d} t+\int_{0}^{T} \int_{\partial \Omega_{s}} D_{s} B_{-s} \delta h-D_{s} \boldsymbol{u}_{s} \cdot \mathbf{w}_{s} \partial_{t} h \mathrm{~d} x \mathrm{~d} y \mathrm{~d} t \\
= & -\int_{0}^{T} \int_{\Omega}\left(\partial_{t}(D \boldsymbol{u})+\nabla \cdot(D \boldsymbol{u} \boldsymbol{u})+\nabla(p+g z)\right) \cdot \mathbf{w}+(D-1) \delta p \mathrm{~d} x \mathrm{~d} y \mathrm{~d} z \mathrm{~d} t \\
& +\int_{0}^{T} \int_{\partial \Omega_{s}} D_{s} B_{-s}\left(\delta h+\mathbf{w}_{s}-\mathbf{w}_{s} \cdot \nabla(h+b)\right)-D_{s} \boldsymbol{u}_{s} \cdot \mathbf{w}_{s}\left(\partial_{t} h+\boldsymbol{v}_{s} \cdot \nabla(h+b)-w_{s}\right) \mathrm{d} x \mathrm{~d} y \mathrm{~d} t
\end{aligned}
$$

where the label displacements are free subject to boundary conditions at fixed side and bottom walls, and where we have used the endpoint condition $\left.\mathbf{w}\right|_{t=0} ^{t=T}=0$ and no normal flow conditions at solid walls. In addition, the function

$B_{-}=\frac{1}{2}|\boldsymbol{u}|^{2}-g z-p$

was used, the outward normal $\hat{\mathbf{n}}_{s}$ at the free surface with surface element $\mathrm{d} s$, and $\hat{\mathbf{n}}_{s} \cdot \boldsymbol{u}_{s} \mathrm{~d} s=\left(w_{s}-\boldsymbol{v}_{s} \cdot \nabla(h+\right.$ b)) $\mathrm{d} x \mathrm{~d} y$. Note that the label displacements $\mathbf{W}$ are free, and directly related to the label variations $\delta \boldsymbol{l}$ (see expression (63) in Appendix A). Condition (9) yields the vanishing of the first boundary term in (11e). The arbitrariness of the variation $\delta p$ yields $D=1$, which together with (6) yields the incompressibility condition, as follows:

$\partial_{t} D+\nabla \cdot(\boldsymbol{u} D)=0$ and $D=1$ together yield $\nabla \cdot \boldsymbol{u}=0$.

The arbitrariness of $\mathbf{w}_{s}$, or $(\delta \boldsymbol{l})_{s}$, yields the kinematic condition (8). The latter follows therefore separately from the variational principle. In turn, the arbitrariness of $\mathbf{w}$ in the interior of the fluid gives via $D=1$ the momentum equations. Hence, we find: 
$\mathbf{w}: \partial_{t} \boldsymbol{u}+\boldsymbol{u} \cdot \nabla \boldsymbol{u}+\nabla(p+g z)=0$ and $\delta p: D=1 \Longrightarrow \nabla \cdot \boldsymbol{u}=0$,

as well as (8). Note that the multiplier $p$ can be interpreted as the pressure field. The incompressibility constraint is maintained in time by taking the time derivative of $\boldsymbol{\nabla} \cdot \boldsymbol{u}=0$ in (13) and imposing this consistency condition, $\partial_{t} \boldsymbol{\nabla} \cdot \boldsymbol{u}=0$, in the gradient of the momentum equation. It leads to a Poisson equation for the pressure $p$.

In this paper, our aim is to develop reduced models that impose a specified vertical profile for the velocity $\boldsymbol{u}$, following the methodology of $[13,21]$, whilst preserving vertical vorticity and the variational structure. In general, the above methodology does not permit the restriction of the vertical profile, unless one assumes that the horizontal components of velocity are depth-independent (columnar motion), in which case one obtains the Green-Naghdi equations [22] (for a description of the above variational process restricted to columnar velocity profiles, see [23], for example). The reason that the Green-Naghdi equations cannot be extended to more general polynomial profiles is that it is not possible to find a subgroup of the group of flow maps (diffeomorphisms) $\chi$ that satisfies (2). In this paper we pursue an alternative direction: we start from an equivalent formulation without the implicit constraints (5), and replace them with the explicit constraint (enforced by Lagrange multipliers) that particle labels (the initial conditions for Lagrangian particles, for example) are advected by the velocity $\boldsymbol{u}$. An additional constraint that the density $D$ satisfies (6) is also required. In [15] and [24], it was shown that formulations of this type are equivalent to formulations of the type described above. For the case of the three-dimensional incompressible Euler equations discussed above, the variational principle becomes

$$
\begin{aligned}
0= & \delta \mathcal{L}[\boldsymbol{l}, \boldsymbol{\pi}, \boldsymbol{u}, p, D, \phi, h, \lambda] \\
= & \delta \int_{0}^{T} \int_{\Omega} \frac{1}{2} D|\boldsymbol{u}|^{2}-g D z+p(1-D)-\underbrace{D \boldsymbol{\pi} \cdot\left(\partial_{t} \boldsymbol{l}+(\boldsymbol{u} \cdot \nabla) \boldsymbol{l}\right)}_{\text {labels term }}+\phi\left(\partial_{t} D+\nabla \cdot(\boldsymbol{u} D)\right) \mathrm{d} x \mathrm{~d} y \mathrm{~d} z \\
& +\int_{\partial \Omega_{s}} \lambda\left(\partial_{t} h+\boldsymbol{v}_{s} \cdot \nabla(h+b)-w_{s}\right) \mathrm{d} x \mathrm{~d} y \mathrm{~d} t,
\end{aligned}
$$

where in conjunction to particle labels $\boldsymbol{l}(\boldsymbol{x}, t), \boldsymbol{\pi}(\boldsymbol{x}, t) \in \mathbb{R}^{3}$ is the vector of Lagrange multipliers constraining the labels to be advected by the velocity field $\boldsymbol{u} ; \phi$ is the Lagrange multiplier that constrains the density $D$ to satisfy equation (6); and, $\lambda$ imposes the kinematic boundary condition (8) as constraint. The kinematic boundary condition (8) can either by explicitly enforced via this Lagrange multiplier $\lambda$, or be retained implicitly. As shown in [24], this variational principle results in the same equations (13). We note that removing the indicated "labels term" from the action principle still results in the same equations but restricts the velocity $\boldsymbol{u}$ to potential (vorticity-free) flow $\boldsymbol{u}=\nabla \phi$ : the labels are required for velocity fields with non-zero vorticity, and a total of three dynamical constraints are required for the most general flows with non-zero helicity. We actually have four constraints in (14), the three components of the labels $\boldsymbol{l}=\left(l_{1}, l_{2}, l_{3}\right)$ and the density equation constraint, which is more than is strictly necessary, but this keeps the formulation tidy.

In Sect.3, we shall see that this potential-flow condition leads to Luke's variational principle for the case of water waves. In Sect. 4 we shall reintroduce vertical vorticity into this formulation by replacing the labels term by a modified constraint that the horizontal components of the particle labels should be advected by the vertically averaged velocity. This allows a model with dispersive non-hydrostatic waves and vertical vorticity.

\section{Luke's variational principle as constrained formulation}

Our aim is to derive water-wave equations with accurate dispersion and vertical vorticity from the parent variational principle (14). The irrotational water-wave equations include full dispersion of free-surface gravity waves. We therefore first (re)derive Luke's variational principle as a constrained formulation from our parent equations. The constraint concerns the potential-flow ansatz for the velocity. Our derivation appears to be novel; more importantly it defines the methodology we use. Luke's principle, however, is only valid for water waves under potential flow, in which there is no vertical vorticity. We lift this restriction later in our new water-wave model. 
We start by removing the labels term from the variational principle (14), and obtain

$$
\begin{aligned}
0= & \delta \int_{0}^{T} \mathcal{L}[\boldsymbol{u}, p, D, \phi, h, \lambda] \mathrm{d} t \\
= & \delta \int_{0}^{T} \int_{\Omega} \frac{1}{2} D|\boldsymbol{u}|^{2}-g D z+p(1-D)+\phi\left(\partial_{t} D+\nabla \cdot(\boldsymbol{u} D)\right) \mathrm{d} x \mathrm{~d} y \mathrm{~d} z \\
& +\int_{\partial \Omega_{s}} \lambda\left(\partial_{t} h+\boldsymbol{v}_{s} \cdot \nabla(h+b)-w_{s}\right) \mathrm{d} x \mathrm{~d} y \mathrm{~d} t .
\end{aligned}
$$

A Legendre transform in $\boldsymbol{u}$ then shows that the potential-flow ansatz follows:

1. use the variational principle to obtain an equation for $\boldsymbol{u}$ and $\lambda$, and then

2. eliminate $\boldsymbol{u}$ and $\lambda$ from the action principle; finally, take variations to obtain the water-wave dynamics.

Taking (unconstrained) variations of (15) in $\boldsymbol{u}$ gives

$$
\begin{aligned}
0 & =\delta \int_{\Omega} \frac{1}{2} D|\boldsymbol{u}|^{2}+\phi \nabla \cdot(D \boldsymbol{u}) \mathrm{d} x \mathrm{~d} y \mathrm{~d} z+\delta \int_{\partial \Omega_{s}} \lambda\left(\partial_{t} h+\boldsymbol{v}_{s} \cdot \nabla(h+b)-w_{s}\right) \mathrm{d} x \mathrm{~d} y \\
& =\int_{\Omega} D \delta \boldsymbol{u} \cdot(\boldsymbol{u}-\nabla \phi) \mathrm{d} x \mathrm{~d} y \mathrm{~d} z+\int_{\partial \Omega_{s}}\left(D_{s} \phi_{s}(\delta \boldsymbol{u})_{s}-\lambda \delta\left(\boldsymbol{u}_{s}\right)\right) \cdot \hat{\boldsymbol{n}}_{s} \mathrm{~d} s
\end{aligned}
$$

and we obtain

$\delta \boldsymbol{u}: \boldsymbol{u}=\nabla \phi \quad$ and $\quad(\delta \boldsymbol{u})_{s}: \lambda=D_{s} \phi_{s}$,

since $\delta\left(\boldsymbol{u}_{s}\right)=(\delta \boldsymbol{u})_{s}+(\partial \boldsymbol{u} / \partial z)_{s} \delta h$. Using this expression, we eliminate $\boldsymbol{u}$ and $\lambda$ from the action principle (15), use Gauss' theorem in space on the term $\phi \nabla \cdot(\boldsymbol{u} D)$, and derive the following

$$
\begin{aligned}
0= & \delta \int_{0}^{T} \mathcal{L}[p, D, \phi, h] \mathrm{d} t \\
= & \delta \int_{0}^{T} \int_{\Omega} \frac{1}{2} D|\nabla \phi|^{2}-g D z+p(1-D)+\phi\left(\partial_{t} D+\nabla \cdot(D \nabla \phi)\right) \mathrm{d} x \mathrm{~d} y \mathrm{~d} z \\
& +\int_{\partial \Omega_{s}} D_{s} \phi_{S}\left(\partial_{t} h+\left(\nabla_{H} \phi\right)_{s} \cdot \nabla(h+b)-\left(\partial_{z} \phi\right)_{s}\right) \mathrm{d} x \mathrm{~d} y \mathrm{~d} t \\
= & \delta \int_{0}^{T} \int_{\Omega}-\frac{1}{2} D|\nabla \phi|^{2}-g D z+p(1-D)+\phi \partial_{t} D \mathrm{~d} x \mathrm{~d} y \mathrm{~d} z+\int_{\partial \Omega_{s}} D_{s} \phi_{s} \partial_{t} h \mathrm{~d} x \mathrm{~d} y \mathrm{~d} t
\end{aligned}
$$

with horizontal gradient $\nabla_{H}=\left(\partial_{x}, \partial_{y}\right)^{T}$. Making use of

$$
\frac{\mathrm{d}}{\mathrm{d} t} \int_{\Omega} D \phi \mathrm{d} x \mathrm{~d} y \mathrm{~d} z=\int_{\Omega} \partial_{t}(D \phi) \mathrm{d} x \mathrm{~d} y \mathrm{~d} z+\int_{\partial \Omega_{S}} \partial_{t} h D_{s} \phi_{s} \mathrm{~d} x \mathrm{~d} y,
$$


variational principle (16) becomes

$$
\begin{aligned}
0 & =\delta \int_{0}^{T} \mathcal{L}[D, \phi, h] \mathrm{d} t \\
& =\delta \int_{0}^{T} \int_{\Omega} D \partial_{t} \phi \mathrm{d} x \mathrm{~d} y \mathrm{~d} z+\mathcal{H} \mathrm{d} t \\
& \equiv \delta \int_{0}^{T} \int_{\Omega} D \partial_{t} \phi+\frac{1}{2} D|\nabla \phi|^{2}+g D z+p(D-1) \mathrm{d} x \mathrm{~d} y \mathrm{~d} z \mathrm{~d} t
\end{aligned}
$$

with Hamiltonian $\mathcal{H}$, provided we consider $\int_{\Omega} D \phi \mathrm{d} x \mathrm{~d} y \mathrm{~d} z$ as a global constant in time. The latter integral constraint determines $\phi$, which would otherwise be fixed up to a constant. Variation of (18) with respect to density $D$ yields the Bernoulli condition

$\delta D: \partial_{t} \phi=-\frac{\delta \mathcal{H}}{\delta D}=-\left(\frac{1}{2}|\nabla \phi|^{2}+g z+p\right)$

throughout the fluid. It defines the pressure. The other variations yield

$$
\begin{aligned}
& \delta p: D=1 \\
& \delta \phi: \partial_{t} D=\frac{\delta \mathcal{H}}{\delta \phi}=-\nabla \cdot(D \nabla \phi), \\
& \delta h:\left(\partial_{t} \phi\right)_{s}=-\frac{\delta \mathcal{H}}{\delta h}=-\left(\frac{1}{2}|\nabla \phi|_{s}^{2}+g(h+b)\right), \\
& (\delta \phi)_{s}: \partial_{t} h=\left(\frac{\delta \mathcal{H}}{\delta \phi}\right)_{s}=\left(\partial_{z} \phi\right)_{s}-\left(\nabla_{H} \phi\right)_{s} \cdot \nabla_{H}(h+b) .
\end{aligned}
$$

Using the constraint $D=1$ in the continuity equation (20b) gives Laplace's equation for $\phi$. The classical waterwave equations thus emerge. Furthermore, by combining Bernoulli's equation at the free surface, $z=h+b$, and the dynamic boundary condition, the boundary condition for the pressure at the free surface explicitly follows as $\left.p\right|_{z=h+b}=0$.

It is also possible to view $\mathbf{w}$ and $\overline{\mathbf{w}}$ defined in (7) and (10) as independent variations rather than $\delta D$ and $\delta h$. Hence, further evaluations using Gauss' and Green's laws are required. The regular momentum equations and equations for free-surface dynamics then emerge:

$$
\begin{aligned}
& \mathbf{w}: D \nabla\left(\partial_{t} \phi+\frac{1}{2}|\nabla \phi|^{2}+g z+p\right)=0, \\
& \overline{\mathbf{w}}: h \nabla_{H}\left(\left(\partial_{t} \phi\right)_{s}+\frac{1}{2}|\nabla \phi|_{s}^{2}+g(h+b)\right)=0 .
\end{aligned}
$$

The former three equations are the momentum equations in potential-flow form; and the latter equations are important for deriving weak solutions for hydraulic jumps and bores as will be discussed later.

Finally, we notice that the incompressibility constraint $\nabla \cdot \boldsymbol{u}=0$ drops out of the formulation if we eliminate the term $p(1-D)$ by substituting $D=1$ in the variational principle. Hence, we obtain Luke's variational principle [17]:

$0=\delta \int_{0}^{T} \mathcal{L}[\phi, h] \mathrm{d} t=\delta \int_{0}^{T} \int_{\Omega} \partial_{t} \phi+\frac{1}{2}|\nabla \phi|^{2}+g z \mathrm{~d} x \mathrm{~d} y \mathrm{~d} z \mathrm{~d} t$, 
which action is the pressure $p$. Variation of (22) gives

$$
\begin{aligned}
0= & \int_{0}^{T}-\int_{\Omega} \nabla^{2} \phi \delta \phi \mathrm{d} x \mathrm{~d} y \mathrm{~d} z+\int_{\partial \Omega_{s}}\left(\partial_{t} \phi+\frac{1}{2}|\nabla \phi|^{2}+g(h+b)\right)_{s} \\
& \times \delta h+\left(w_{s}-\partial_{t} h-(\nabla \phi)_{s} \cdot \nabla(h+b)\right)(\delta \phi)_{s} \mathrm{~d} x \mathrm{~d} y \mathrm{~d} t .
\end{aligned}
$$

These lead to the "classical" water-wave equations under the assumption of potential flow:

$$
\begin{aligned}
& \delta \phi: \nabla^{2} \phi=0, \\
& \delta h:\left(\partial_{t} \phi\right)_{s}+\frac{1}{2}|\nabla \phi|_{s}^{2}+g(h+b)=0, \\
& (\delta \phi)_{s}: \partial_{t} h+(\nabla \phi)_{s} \cdot \nabla(h+b)-\left(\partial_{z} \phi\right)_{s}=0 .
\end{aligned}
$$

This justifies the substitution of $D=1$ a posteriori, but without provision of Bernoulli's equation (19) in the interior. Therefore, Luke's principle does not provide an explicit expression for $p$, even though we indirectly know its action to be the pressure.

Luke's variational principle can be rewritten in another form by using (17) with $D=1$, leading to Miles' variational principle [18]:

$$
0=\delta \int_{0}^{T} \mathcal{L}[\phi, h] \mathrm{d} t=\delta \int_{0}^{T} \int_{\Omega} \frac{1}{2}|\nabla \phi|^{2}+g z \mathrm{~d} x \mathrm{~d} y \mathrm{~d} z-\int_{\partial \Omega_{s}} \phi_{s} \partial_{t} h \mathrm{~d} x \mathrm{~d} y \mathrm{~d} t .
$$

\section{Constrained variational principle: water waves with vertical vorticity}

\subsection{Variational principles}

Our next step is to simplify the labels constraint and derive a new water-wave model with both the irrotational water-wave model and the depth-averaged shallow-water equations as limiting forms. We therefore limit the labels to be horizontal $\boldsymbol{l}(x, y, t) \in \mathbb{R}^{2}$ and from the onset constrain these to depend on the horizontal coordinates only. This contrasts with the general case considered in Sect. 2. In essence, the resulting labels are advected by a depth and density-weighted horizontal velocity, that is

$$
\frac{1}{h} \int_{b}^{h+b} D \mathrm{~d} z \partial_{t} \boldsymbol{l}+\frac{1}{h} \int_{b}^{h+b} D \boldsymbol{u} \mathrm{d} z \cdot \nabla \boldsymbol{l}=0 .
$$

Similar to the fully 3D formulation in Sect. 2, we shall see that adding the constraint that both components of the labels are advected by the vertically averaged velocity field gives rise to an overdetermined system. However, as we shall describe, this is more convenient than only constraining one component. Throughout we use slip-flow boundary conditions at fixed walls.

After multiplication by depth $h$, these advected constrained labels are enforced by additional Lagrange multipliers $\pi=\pi(x, y, t) \in \mathbb{R}^{2}$, also independent of $z$. We anticipate that $D$ can be set to unity later, given our derivation of Luke's principle. Hence, the variational principle, either (14) or (15), is changed to

$0=\delta \int_{0}^{T} \int_{\Omega} \frac{1}{2} D|\boldsymbol{u}|^{2}-g D z+p(1-D)+\phi\left(\partial_{t} D+\nabla \cdot(\boldsymbol{u} D)\right)-D \boldsymbol{\pi} \cdot\left(\partial_{t} \boldsymbol{l}+\boldsymbol{u} \cdot \nabla \boldsymbol{l}\right) \mathrm{d} x \mathrm{~d} y \mathrm{~d} z \mathrm{~d} t$.

This time we chose to include the kinematic condition (8) implicitly instead of explicitly, but either route suffices. A similar calculation as before for the $\boldsymbol{u}$-variation yields

$\boldsymbol{u} \equiv \nabla \phi(x, y, z, t)+\mathbf{v}(x, y, t) \equiv \nabla \phi(x, y, z, t)+(\nabla \boldsymbol{l}(x, y, t))^{T} \boldsymbol{\pi}(x, y, t)$,

adopting the notation 


$$
\left[(\nabla \boldsymbol{l})^{T} \pi\right]_{i j}=\left(\partial_{x_{i}} l_{j}\right) \pi_{j}
$$

Such a splitting does not uniquely determine $\nabla \phi$, since $v$ can in general have a non-zero divergent component. This degeneracy can be removed by only including the constraint for one component $l_{1}$ of the labels $\boldsymbol{l}$, but the use of two labels ensures that $\mathbf{v}$ can point in any (horizontal) direction. In the following, we shall see that we retain $\mathbf{v}$ in the reduced form, which determines $\phi$ exactly from a Poisson equation.

We substitute (28) in action principle (27) to get:

$$
\begin{aligned}
0 & =\delta \int_{0}^{T} \mathcal{L}[\boldsymbol{l}, \boldsymbol{\pi}, p, D, \phi, h] \mathrm{d} t=\delta \int_{0}^{T} \int_{\Omega} \frac{1}{2} D|\nabla \phi+\mathbf{v}|^{2}-g D z+p(1-D)+\phi\left(\partial_{t} D+\nabla \cdot(D(\nabla \phi+\mathbf{v}))\right) \\
& -D \pi \cdot\left(\partial_{t} \boldsymbol{l}+(\nabla \phi+\mathbf{v}) \cdot \nabla \boldsymbol{l}\right) \mathrm{d} x \mathrm{~d} y \mathrm{~d} z \mathrm{~d} t \\
& =-\delta \int_{0}^{T} \int_{\Omega} \frac{1}{2} D|\nabla \phi+\mathbf{v}|^{2}+g D z+p(D-1)+D \pi \cdot \partial_{t} \boldsymbol{l}-\phi \partial_{t} D \mathrm{~d} x \mathrm{~d} y \mathrm{~d} z+\int D_{s} \phi_{s} \partial_{t} h \mathrm{~d} x \mathrm{~d} y \mathrm{~d} t
\end{aligned}
$$

in which the vector $\mathbf{v}$ lies in the $x-y$-plane and is a shorthand defined in (28b) and not an independent variable. Making use of (17) and the corresponding integral constraint, and then changing the sign of the variational principle yields

$$
\begin{aligned}
0 & =\delta \int_{0}^{T} \mathcal{L}[\boldsymbol{l}, \boldsymbol{\pi}, D, \phi, h] \mathrm{d} t \\
& =\delta \int_{0}^{T} \int_{\Omega} D \partial_{t} \phi+D \pi \cdot \partial_{t} \boldsymbol{l}+\frac{1}{2} D|\nabla \phi+\mathbf{v}|^{2}+g D z+p(D-1) \mathrm{d} x \mathrm{~d} y \mathrm{~d} z \mathrm{~d} t \\
& \equiv \delta \int_{0}^{T} \int_{\Omega} D \partial_{t} \phi+D \pi \cdot \partial_{t} \boldsymbol{l} \mathrm{d} x \mathrm{~d} y \mathrm{~d} z+\mathcal{H} \mathrm{d} t
\end{aligned}
$$

which defines the Hamiltonian $\mathcal{H}$. Variation of (30) yields:

$\delta D: \partial_{t} \phi=-\frac{\delta \mathcal{H}}{\delta D}=-\left(\frac{1}{2}|\nabla \phi+\mathbf{v}|^{2}+g z+p-\int_{b}^{b+h} D(\nabla \phi+\mathbf{v}) \mathrm{d} z \cdot \mathbf{v} /(h \bar{D})\right)$,

$\delta \phi: \partial_{t} D=\frac{\delta \mathcal{H}}{\delta \phi}=-\nabla \cdot(D(\nabla \phi+\mathbf{v}))$

$\delta h: D_{s}\left(\partial_{t} \phi\right)_{s}=-\frac{\delta \mathcal{H}}{\delta h}=-\left(\frac{1}{2} D|\nabla \phi+\mathbf{v}|^{2}+g D(h+b)+p(D-1)-D_{s} \int_{b}^{b+h} D(\nabla \phi+\mathbf{v}) \mathrm{d} z \cdot \mathbf{v} /(h \bar{D})\right)_{s}$,

$(\delta \phi)_{s}: D_{s} \partial_{t} h=\left(\frac{\delta \mathcal{H}}{\delta \phi}\right)_{s}=D_{s}\left(w_{s}-\boldsymbol{v}_{s} \cdot \nabla(h+b)\right)$,

$\delta(h \bar{D} \pi): \partial_{t} \boldsymbol{l}=-\frac{\delta \mathcal{H}}{\delta(h \bar{D} \pi)}=-\left(\int_{b}^{b+h} D(\nabla \phi+\mathbf{v}) \mathrm{d} z \cdot \nabla \boldsymbol{l}\right) /(h \bar{D})$, 
$\delta \boldsymbol{l}: \partial_{t}(h \bar{D} \boldsymbol{\pi})=\frac{\delta \mathcal{H}}{\delta \boldsymbol{l}}=-\nabla \cdot\left(\int_{b}^{b+h} D(\nabla \phi+\mathbf{v}) \mathrm{d} z \boldsymbol{\pi}\right)$,

$\delta p: D-1=0$.

This includes an extended Bernoulli's relation for the variation with respect to $D$. The variation is chosen with respect to $h \bar{D} \pi$ with $h \bar{D}=\int_{b}^{b+h} D \mathrm{~d} z$ to obtain (nearly) canonical equations. Finally, we include incompressibility by setting $D=1$ in (30) to obtain the extended variational principle

$$
\begin{aligned}
0 & =\delta \int_{0}^{T} \mathcal{L}[\boldsymbol{l}, \boldsymbol{\pi}, \phi, h] \mathrm{d} t \\
& =\delta \int_{0}^{T} \int_{\Omega} \partial_{t} \phi+\frac{1}{2}|\nabla \phi+\mathbf{v}|^{2} \mathrm{~d} x \mathrm{~d} y \mathrm{~d} z+\int_{\partial \Omega_{s}} h \boldsymbol{\pi} \cdot \partial_{t} l+\frac{1}{2} g\left((h+b)^{2}-b^{2}\right) \mathrm{d} x \mathrm{~d} y \mathrm{~d} t .
\end{aligned}
$$

Variation of (32) gives

$$
\begin{aligned}
& 0=\int_{0}^{T} \int_{\Omega}(\nabla \phi+\mathbf{v}) \cdot(\nabla \delta \phi+\delta \mathbf{v})+\partial_{t} \delta \phi \mathrm{d} x \mathrm{~d} y \mathrm{~d} z \\
& +\int_{0}^{T} \int_{\partial \Omega_{S}}\left(\partial_{t} \phi+\frac{1}{2}|\nabla \phi+\mathbf{v}|^{2}+g(h+b)+\boldsymbol{\pi} \cdot \partial_{t} \boldsymbol{l}\right)_{s} \delta h+h \delta \boldsymbol{\pi} \cdot \partial_{t} \boldsymbol{l}+h \boldsymbol{\pi} \cdot \partial_{t} \delta \boldsymbol{l} \mathrm{d} x \mathrm{~d} y \mathrm{~d} t \\
& =-\int_{0}^{T} \int_{\Omega}\left(\nabla^{2} \phi+\nabla \cdot \mathbf{v}\right) \delta \phi \mathrm{d} x \mathrm{~d} y \mathrm{~d} z+\left.\int_{\Omega} \delta \phi\right|_{t=0} ^{t=T} \mathrm{~d} x \mathrm{~d} y \mathrm{~d} z \\
& +\int_{0}^{T} \int_{\partial \Omega_{s}}\left(\partial_{t} \phi+\frac{1}{2}|\nabla \phi+\mathbf{v}|^{2}+g(h+b)+\pi \cdot \partial_{t} \boldsymbol{l}\right)_{s} \delta h+h \delta \boldsymbol{\pi} \cdot \partial_{t} \boldsymbol{l}+h \boldsymbol{\pi} \cdot \partial_{t} \delta \boldsymbol{l} \\
& +(\delta \phi)_{s}\left(\boldsymbol{u}_{s} \cdot\left(-\nabla_{H}(h+b), 1\right)^{T}-\partial_{t} h\right)+h \overline{\boldsymbol{u}} \cdot \delta \mathbf{v} \mathrm{d} x \mathrm{~d} y \mathrm{~d} t \\
& =-\int_{0}^{T} \int_{\Omega}\left(\nabla^{2} \phi+\nabla \cdot \mathbf{v}\right) \delta \phi \mathrm{d} x \mathrm{~d} y \mathrm{~d} z+\int_{0}^{T} \int_{\partial \Omega_{s}}\left(\partial_{t} \phi+\frac{1}{2}|\nabla \phi+\mathbf{v}|^{2}+g(h+b)+\boldsymbol{\pi} \cdot \partial_{t} l\right)_{s} \delta h \\
& +h \delta \boldsymbol{\pi} \cdot\left(\partial_{t} \boldsymbol{l}+\overline{\boldsymbol{u}} \cdot \nabla \boldsymbol{l}\right)-\left(\partial_{t}(h \boldsymbol{\pi})+\nabla \cdot(h \overline{\boldsymbol{u}} \boldsymbol{\pi})\right) \cdot \delta \boldsymbol{l}+(\delta \phi)_{s}\left(w_{s}-\boldsymbol{v}_{s} \cdot \nabla(h+b)-\partial_{t} h\right) \mathrm{d} x \mathrm{~d} y \mathrm{~d} t \\
& +\left.\int_{\Omega_{S}} h \boldsymbol{\pi} \cdot \delta \boldsymbol{l}\right|_{t=0} ^{t=T} \mathrm{~d} x \mathrm{~d} y,
\end{aligned}
$$

where the horizontal (rotational) velocity vector $\mathbf{v}$ and the depth-averaged horizontal velocity vector $\overline{\boldsymbol{u}}$ were used:

$\mathbf{v}=(\nabla \boldsymbol{l})^{T} \boldsymbol{\pi}$ and $h \overline{\boldsymbol{u}}=\int_{b}^{b+h} \boldsymbol{u}_{H} \mathrm{~d} z$ 
End-point conditions used are $\left.\delta \boldsymbol{l}\right|_{t=0} ^{t=T}=0$ and $\left.\delta \phi\right|_{t=0} ^{t=T}=0$, whence terms involving these expressions in (33) vanish. The dynamics arising from (33) are therefore

$$
\begin{aligned}
& \delta \phi: \nabla^{2} \phi+\nabla \cdot \mathbf{v}=0, \\
& \delta h:\left(\partial_{t} \phi\right)_{s}+\frac{1}{2}|\nabla \phi+\mathbf{v}|_{s}^{2}+g(h+b)+\boldsymbol{\pi} \cdot \partial_{t} \boldsymbol{l}=0, \\
& \delta \boldsymbol{\pi}: \partial_{t} \boldsymbol{l}+\overline{\boldsymbol{u}} \cdot \nabla \boldsymbol{l}=0, \\
& (\delta \phi)_{s}:\left(\partial_{z} \phi\right)_{s}-\left(\left(\nabla_{H} \phi\right)_{s}+\mathbf{v}\right) \cdot \nabla_{H}(h+b)-\partial_{t} h=0, \\
& \delta \boldsymbol{l}: \partial_{t}(h \boldsymbol{\pi})+\nabla \cdot(h \overline{\boldsymbol{u}} \boldsymbol{\pi})=0,
\end{aligned}
$$

together with (34). The system (35) equals system (31), after excluding the extended Bernoulli equation in the interior of the fluid. It justifies, a posteriori, the direct substitution of constraint $D=1$ in the variational principle. The above system can be expressed in terms of $\mathbf{v}$, instead of $\boldsymbol{\pi}$ and $\boldsymbol{l}$, by multiplication of (35c) by $\boldsymbol{\pi}$ and elimination of $\boldsymbol{\pi} \cdot \partial_{t} l$ in (35b), and by evaluation of $\partial_{t}(h \overline{\mathbf{v}})$. In addition, we can rewrite (35d). The reformulated system becomes more familiar and reads

$$
\begin{aligned}
& \nabla^{2} \phi+\nabla \cdot \mathbf{v}=0, \\
& \left(\partial_{t} \phi\right)_{s}+\frac{1}{2}\left|(\nabla \phi)_{s}+\mathbf{v}\right|^{2}+g(h+b)-\mathbf{v} \cdot \overline{\boldsymbol{u}}=0, \\
& \partial_{t} h+\nabla \cdot(h \overline{\boldsymbol{u}})=0, \\
& \partial_{t}(h \mathbf{v})+\nabla \cdot(h \overline{\boldsymbol{u}} \mathbf{v})+h \mathbf{v} \nabla \overline{\boldsymbol{u}}=0, \\
& h \overline{\boldsymbol{u}}=\int_{b}^{b+h} \nabla_{H} \phi \mathrm{d} z+h \mathbf{v} .
\end{aligned}
$$

It is seen to be a modification of the water-wave equations under the potential-flow condition; it includes a component of the horizontal momentum $h \mathbf{v}$ which has a layer-averaged vertical component of the vorticity. ${ }^{1}$

Momentum equations for the depth-averaged flow emerge by manipulating (36). Taking the sum of the horizontal gradient of (36b), multiplied thereafter by $h$, and (36d), while using definition (36e), gives:

$$
\partial_{t}(h \overline{\boldsymbol{u}})+\nabla \cdot\left(h \overline{\boldsymbol{u}} \overline{\boldsymbol{u}}+\frac{1}{2} g h^{2}\right)+g h \boldsymbol{\nabla} b+\underline{h\left(\nabla\left(\partial_{t} \phi\right)_{s}-\partial_{t} \overline{\nabla_{H} \phi}\right)}+\underline{\frac{1}{2}} h \boldsymbol{\nabla}\left(\left|\boldsymbol{u}_{S}\right|^{2}-|\overline{\boldsymbol{u}}|^{2}\right)+h\left(\overline{\boldsymbol{u}} \nabla \overline{\nabla_{H} \phi}-\overline{\boldsymbol{u}} \cdot \nabla \overline{\nabla_{H} \phi}\right)=0
$$

with the depth-averaged horizontal velocity vector $\overline{\boldsymbol{u}}$, the three-dimensional velocity vector $\boldsymbol{u}_{S}$ evaluated at the free surface, velocity potential $\phi$, depth $h$, and $\overline{\nabla_{H} \phi} \equiv(1 / h) \int_{b}^{b+h} \nabla_{H} \phi \mathrm{d} z$. The last two terms concern a rotational component, which is nonzero due to the averaging. The underline indicates terms extra compared to the depth-averaged shallow-water equations.

\subsection{Conservation laws}

Following the methodology described in [24], we note that the variational principle (32) has relabelling symmetries of the type:

$$
\delta \boldsymbol{l}=\boldsymbol{\xi}(\boldsymbol{l}), \quad \delta \boldsymbol{\pi}=-(\nabla \boldsymbol{\xi}(\boldsymbol{l}))^{T} \boldsymbol{\pi},
$$

for any smooth vector field $\boldsymbol{\xi}$. These are the infinitesimal symmetries obtained from transformations $\boldsymbol{l} \mapsto \boldsymbol{\phi}(\boldsymbol{l})$, where $\phi$ is a relabelling diffeomorphism (change of coordinates) of the horizontal domain.

Noether's theorem then implies the conservation law

$$
\frac{\partial}{\partial t}(h \boldsymbol{\pi} \cdot \boldsymbol{\xi}(\boldsymbol{l}))+\nabla \cdot(\overline{\boldsymbol{u}} \boldsymbol{\pi} \cdot \boldsymbol{\xi}(\boldsymbol{l}) h)=0,
$$

for the solutions of (36). When this is combined with the continuity equation (36c), we obtain

\footnotetext{
${ }^{1}$ Following choices in classical water wave modeling, the choice $\phi=0$ at the free surface could deal with the overdetermined character of the system at the free surface.
} 


$$
\left(\frac{\partial}{\partial t}+\overline{\boldsymbol{u}} \cdot \nabla\right)(\boldsymbol{\pi} \cdot \boldsymbol{\xi}(\boldsymbol{l}))=0 .
$$

Following [15,24], if we choose a closed loop $C(t)$ in the horizontal plane, advected by the horizontally averaged velocity $\overline{\boldsymbol{u}}$, and take $\boldsymbol{\xi}$ to be tangent to the loop at $t=0$ with $|\boldsymbol{\xi}|=1$, then

$\xi \cdot \mathrm{d} \boldsymbol{x}=(\nabla \boldsymbol{l}) \cdot \mathrm{d} \boldsymbol{x}$

for all times. Hence, we obtain the horizontal circulation-conservation law

$$
\frac{\mathrm{d}}{\mathrm{d} t} \oint_{C(t)}((\boldsymbol{\pi} \cdot \nabla) \boldsymbol{l}) \cdot \mathrm{d} \boldsymbol{x}=0 .
$$

Recalling that $\mathbf{v}=\boldsymbol{\pi} \cdot \nabla \boldsymbol{l}$, Stokes' theorem gives a potential vorticity equation for the total velocity $\boldsymbol{u}=\nabla \phi+\mathbf{v}$ instead of the depth-averaged one:

$\partial_{t} q+\overline{\boldsymbol{u}} \cdot \nabla q=0$

with potential vorticity $q=\nabla \times \boldsymbol{u} / h=\nabla \times \mathbf{v} / h$. Thus (32) describes the vertical vorticity advected by the vertically-averaged velocity inside the fluid, coupled to $z$-dependent potential flow in the interior of the fluid, and connected to the free surface. The variational principle (32) is also invariant under translations in time, and so it has a locally conserved energy $|\boldsymbol{u}|^{2} / 2+g z$.

\subsection{Water-wave and shallow-water limit}

The system (36) reduces to the water-wave equations under potential flow (24) when we either take $\mathbf{v}=0$ or $\mathbf{v}=\nabla_{H} \varphi$. In the latter case, the equation for the time evolution of $\mathbf{v}$ can be written as the horizontal gradient of a Bernoulli-type function. This then recombines with the dynamic condition at the free surface such that the total potential becomes $\phi+\varphi$. Replacement of $\phi+\varphi$ by $\phi$ notationally then yields the desired result, and similarly Poisson's equation reduces to Laplace's equation.

The shallow-water limit is reached when we restrict $\phi=\phi(x, y, z, t)$ to $\phi_{s}=\phi_{s}(x, y, t)$ in the variational principle (32). The result is:

$\partial_{t} \phi_{s}+\frac{1}{2}|\overline{\boldsymbol{u}}|^{2}+g(h+b)+\boldsymbol{\pi} \cdot \partial_{t} \boldsymbol{l}=0$,

$\partial_{t} \boldsymbol{l}+\overline{\boldsymbol{u}} \cdot \nabla \boldsymbol{l}=0$,

$-\nabla_{H} \cdot(h \overline{\boldsymbol{u}})-\partial_{t} h=0$,

$\partial_{t}(h \boldsymbol{\pi})+\nabla \cdot(h \overline{\boldsymbol{u}} \boldsymbol{\pi})=0$.

This shallow-water system can be rewritten as

$\partial_{t} h+\nabla \cdot(h \overline{\boldsymbol{u}})=0$,

$\partial_{t} \phi_{s}+\frac{1}{2}|\overline{\boldsymbol{u}}|^{2}+g(h+b)-\mathbf{v} \cdot \overline{\boldsymbol{u}}=0$,

$\partial_{t} \mathbf{v}+\overline{\boldsymbol{u}} \cdot \nabla \mathbf{v}+\mathbf{v} \nabla \overline{\boldsymbol{u}}=0$,

$\overline{\boldsymbol{u}}=\nabla_{H} \phi_{S}+\mathbf{v}$.

The time derivative of $\overline{\boldsymbol{u}}$ then gives the depth-averaged shallow-water momentum equation

$\partial_{t} \overline{\boldsymbol{u}}+\overline{\boldsymbol{u}} \cdot \nabla \overline{\boldsymbol{u}}+g \nabla(h+b)=0$.

Alternatively, note that the last (underlined) terms in (37) vanish in the shallow-water limit, when $\boldsymbol{u}$ and $\phi$ evaluated at the surface equal their depth-averaged counterparts, whence (37) becomes the regular shallow-water momentum equation in nearly conservative form. 
Fig. 5 Sketch of a vertical cross-section of the fluid's domain showing the surface of an overturned wave and its bore approximation

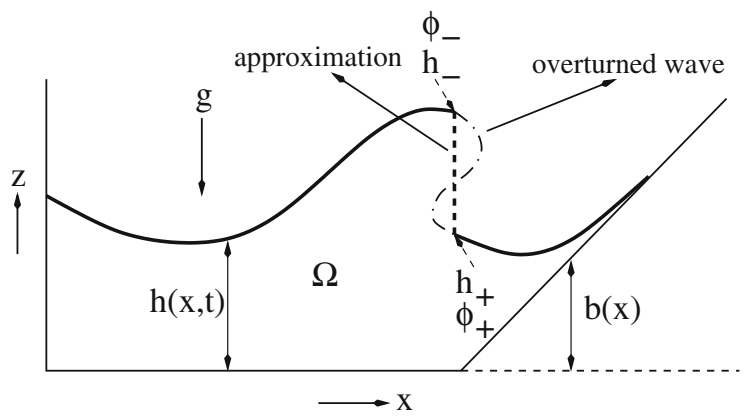

\subsection{Weak formulation: extended hydraulic jump relations}

A weak formulation is sought such that overturning of the free surface is approximated by a local discontinuity. In the shallow-water limit the resulting hydraulic jump or bore relations across the discontinuity should reduce to those for the shallow-water equations. Consequently, the depth $h$ and the depth-averaged velocity will be single-valued except for local line discontinuities in the horizontal. In addition, a new boundary condition for $\phi$ is required for the elliptical equation (36a) along the jump. A sketch of a domain with an overturning wave and the approximation with one jump is given in Fig. 5.

The candidate equations for the weak formulation followed by rewriting (35) into:

$$
\begin{aligned}
& \partial_{t} h+\nabla \cdot(h \overline{\boldsymbol{u}})=0, \\
& \partial_{t}(h \overline{\boldsymbol{u}})+\nabla \cdot\left(h \overline{\boldsymbol{u}} \overline{\boldsymbol{u}}+\frac{1}{2} g h^{2}\right)+g h \nabla b+\underline{h\left(\nabla\left(\partial_{t} \phi\right)_{s}-\partial_{t} \overline{\nabla_{H} \phi}\right)} \\
& \quad+\frac{1}{2} h \nabla_{H}\left(\left|\boldsymbol{u}_{s}\right|^{2}-|\overline{\boldsymbol{u}}|^{2}\right)+h\left(\overline{\boldsymbol{u}} \nabla \overline{\nabla_{H} \phi}-\overline{\boldsymbol{u}} \cdot \nabla \overline{\nabla_{H} \phi}\right)=0
\end{aligned}
$$

with the depth-averaged horizontal velocity vector $\overline{\boldsymbol{u}}$, the three-dimensional velocity vector $\boldsymbol{u}_{s}$ evaluated at the free surface, velocity potential $\phi$, and depth $h$. There is a theory for hyperbolic systems with nonconservative products [25] and numerical applications thereof $[26,27]$. The term $h \nabla b$ is an example of an intrinsic nonconservative product, also when we consider the shallow-water limit. This matters when $b$ contains jumps, either in its definition or its numerical approximation. It is intrinsic because the term cannot be brought in conservative form without destroying the conservative structure in the remaining shallow-water momentum equations. The underlined terms are also nonconservative terms but the theory of Dal Maso [25] does not directly apply, because our extended water-wave system is a coupled elliptic-hyperbolic system. The theory of hyperbolic systems with nonconservative products shows that the extended Rankine-Hugoniot or shock relations depend on the path chosen in the calculation. Besides the strict nonapplicability of this theory for our coupled elliptic-hyperbolic system, the choice of path is open. In many cases, Rhebergen et al. [27] show numerically that a linear path suffices as a reasonable choice.

Without a derivation of an extended theory for elliptic-hyperbolic systems and non-conservative products, two approximations may be reasonable. First, the additional underlined terms in (47b) are ignored at leading order, and the jump relations for the shallow-water equations can be used as a crude approximation. Second, this theory of nonconservative products can be applied in a heuristic manner while using a linear path. In simplified terms, generalized Rankine-Hugoniot relations emerge by consideration of (47) in space time. Upon using Gauss' theorem in horizontal space time, the weak formulation across the shock surface in space time amounts to [27]:

$$
-S_{n} \int_{0}^{1} \partial_{\tau} \tilde{h}\left(\tau ; U^{-}, U^{+}\right) \mathrm{d} \tau+\int_{0}^{1} \partial_{\tau} \widetilde{(h \overline{\boldsymbol{u}})}\left(\tau ; U^{-}, U^{+}\right) \cdot \hat{\mathbf{n}} \mathrm{d} \tau
$$

for the continuity equation where we use a vector $\left(-S_{n}, n_{x}, n_{y}\right)^{T}$ parallel to the space time normal vector, the space normal $\hat{\mathbf{n}}=\left(n_{x}, n_{y}\right)^{T}$ in the horizontal plane, and the shock speed $S_{n}$. The variables with a tilde depend on the 
states of the variables, denoted by $U^{-}, U^{+}$across the shock, on $\tau$, and the path between $\tau=0$ and $\tau=1$, with $U=(h, h \overline{\boldsymbol{u}}, b, \phi)$. Furthermore, $\tilde{h}\left(0 ; U^{-}, U^{+}\right)=h^{-}$and $\tilde{h}\left(1 ; U^{-}, U^{+}\right)=h^{+}$, and so forth. However, due to its conservative form expression (48) is immediately integrable and leads to the classic jump relation, expressing mass conservation across the shock:

$\left[h\left(\overline{\boldsymbol{u}} \cdot \hat{\mathbf{n}}-S_{n}\right)\right]=0$

with $[U]=U^{+}-U^{-}$denoting the jump across. Similarly, for the momentum equations we find:

$$
\begin{aligned}
& -S_{n} \int_{0}^{1} \partial_{\tau} \widetilde{(h \overline{\boldsymbol{u}})}\left(\tau ; U^{-}, U^{+}\right) \mathrm{d} \tau+\int_{0}^{1} \partial_{\tau}\left(h \overline{\boldsymbol{u}} \overline{\boldsymbol{u}}+\frac{1}{2} g h^{2}\right)\left(\tau ; U^{-}, U^{+}\right) \hat{\mathbf{n}} \mathrm{d} \tau \\
& +\int_{0}^{1} g \tilde{h}\left(\tau ; U^{-}, U^{+}\right) \partial_{\tau} \tilde{b}\left(\tau ; U^{-}, U^{+}\right) \hat{\mathbf{n}} \mathrm{d} \tau+S_{n} \int_{0}^{1} \tilde{h}\left(\tau ; U^{-}, U^{+}\right) \partial_{\tau} \widetilde{\nabla_{H} \phi}\left(\tau ; U^{-}, U^{+}\right) \mathrm{d} \tau \\
& +\int_{0}^{1} \tilde{h}\left(\tau ; U^{-}, U^{+}\right) \partial_{\tau} \widetilde{\left(\partial_{t} \phi\right)_{S}}\left(\tau ; U^{-}, U^{+}\right) \hat{\mathbf{n}} \mathrm{d} \tau+\frac{1}{2} \int_{0}^{1} \tilde{h}\left(\tau ; U^{-}, U^{+}\right) \partial_{\tau}\left(\left|\boldsymbol{u}_{s}\right|^{2}-|\overline{\boldsymbol{u}}|^{2}\right)\left(\tau ; U^{-}, U^{+}\right) \hat{\mathbf{n}} \mathrm{d} \tau \\
& +\int_{0}^{1}\left(\widetilde{h \overline{\boldsymbol{u}}}\left(\tau ; U^{-}, U^{+}\right) \partial_{\tau} \widetilde{\overline{\nabla_{H} \phi}}\left(\tau ; U^{-}, U^{+}\right)-\widetilde{\bar{h}}\left(\tau ; U^{-}, U^{+}\right) \cdot \partial_{\tau} \widetilde{\overline{\nabla_{H} \phi}}\right) \hat{\mathbf{n}} \mathrm{d} \tau \\
& =\left[h \overline{\boldsymbol{u}}\left(\overline{\boldsymbol{u}} \cdot \hat{\mathbf{n}}-S_{n}\right)\right]+\left[\frac{1}{2} g h^{2}\right] \hat{\mathbf{n}} \\
& +\int_{0}^{1} g \tilde{h}\left(\tau ; U^{-}, U^{+}\right) \partial_{\tau} \tilde{b}\left(\tau ; U^{-}, U^{+}\right) \hat{\mathbf{n}} \mathrm{d} \tau+S_{n} \int_{0}^{1} \tilde{h}\left(\tau ; U^{-}, U^{+}\right) \partial_{\tau} \widetilde{\nabla_{H} \phi}\left(\tau ; U^{-}, U^{+}\right) \mathrm{d} \tau \\
& +\int_{0}^{1} \tilde{h}\left(\tau ; U^{-}, U^{+}\right) \partial_{\tau} \widetilde{\left(\partial_{t} \phi\right)_{S}}\left(\tau ; U^{-}, U^{+}\right) \hat{\mathbf{n}} \mathrm{d} \tau+\frac{1}{2} \int_{0}^{1} \tilde{h}\left(\tau ; U^{-}, U^{+}\right) \partial_{\tau}\left(\left|\boldsymbol{u}_{S}\right|^{2}-|\overline{\boldsymbol{u}}|^{2}\right)\left(\tau ; U^{-}, U^{+}\right) \hat{\mathbf{n}} \mathrm{d} \tau \\
& +\int_{0}^{1}\left(\widetilde{h \overline{\boldsymbol{u}}}\left(\tau ; U^{-}, U^{+}\right) \partial_{\tau} \widetilde{\widetilde{\nabla_{H} \phi}}\left(\tau ; U^{-}, U^{+}\right)-\widetilde{h \overline{\boldsymbol{u}}}\left(\tau ; U^{-}, U^{+}\right) \cdot \partial_{\tau} \widetilde{\widetilde{\nabla_{H} \phi}}\left(\tau ; U^{-}, U^{+}\right)\right) \hat{\mathbf{n}} \mathrm{d} \tau=0 .
\end{aligned}
$$

Note that we have integrated by parts in $\tau$ for some terms, such that $\int_{0}^{1} h \partial_{\tau} Q \mathrm{~d} \tau=[h Q]-\int_{0}^{1}[h] Q \mathrm{~d} \tau$ for some function $Q$ depending on the variables, and that a linear path is chosen for $h$ and the product $h \overline{\boldsymbol{u}}$. The first terms are conservative and integrable for any path function, and lead to the well-known jump condition for the shallow-water momentum equations, while we need to choose or determine a path for the remaining terms. We choose a linear path $U=U^{-}+\tau\left(U^{+}-U^{-}\right)$based on the generally weak path dependence observed in [27]. Hence, we obtain the approximated jump relation for our extended system:

$$
\begin{aligned}
& {\left[h \overline{\boldsymbol{u}}\left(\overline{\boldsymbol{u}} \cdot \hat{\mathbf{n}}-S_{n}\right)\right]+\left[\frac{1}{2} g h^{2}+g\{h\} b\right] \hat{\mathbf{n}}+\left[S_{n} h \overline{\nabla_{H} \phi}+h\left(\partial_{t} \phi\right)_{s}+\frac{1}{2} h\left(\left|\boldsymbol{u}_{S}\right|^{2}-|\overline{\boldsymbol{u}}|^{2}\right)\right.} \\
& \left.+h\left(\overline{\boldsymbol{u}} \overline{\nabla_{H} \phi}-\overline{\boldsymbol{u}} \cdot \overline{\nabla_{H} \phi}\right)\right] \hat{\mathbf{n}} \\
& \quad-\int_{0}^{1}\left\{S_{n}[h] \widetilde{\widetilde{\nabla_{H} \phi}}\left(\tau ; U^{-}, U^{+}\right)+[h] \widetilde{\left(\partial_{t} \phi\right)_{S}}\left(\tau ; U^{-}, U^{+}\right)\right. \\
& \left.\quad+\frac{1}{2}[h]\left(\mid \boldsymbol{u}_{S} \widetilde{\left.\right|^{2}-|\overline{\boldsymbol{u}}|^{2}}\right)\left(\tau ; U^{-}, U^{+}\right)+\left([h \overline{\boldsymbol{u}}] \widetilde{\bar{\nabla}_{H} \phi}\left(\tau ; U^{-}, U^{+}\right)-[h \overline{\boldsymbol{u}}] \cdot \widetilde{\nabla_{H} \phi}\left(\tau ; U^{-}, U^{+}\right)\right)\right\} \hat{\mathbf{n}} \mathrm{d} \tau=0
\end{aligned}
$$


with mean $\{U\}=\left(U^{-}+U^{+}\right) / 2$ and $[\{U\} b]=\{U\}[b]$. The last term with $|\overline{\boldsymbol{u}}|^{2}$ can be integrated directly, but the others need to be determined numerically or approximated further, e.g., by using a linear path in $\tau$ for $\boldsymbol{u}_{s},\left(\partial_{t} \phi\right)_{s}$, $(1 / h) \int_{b}^{b+h} \nabla_{H} \phi \mathrm{d} z$, et cetera. When we approximate $[h]=[H]$ for some (constant) mean depth $H$ when the topography is flat, then the last terms disappear. The momentum equations are conservative in this (inconsistent) small amplitude limit, which merely illustrates that the remaining terms are as expected.

In a vertical plane the new water-wave model has no circulation, since $\left(u_{1}, u_{3}\right)=\left(\partial_{x} \phi+\mathrm{v}(x, y, t), \partial_{z} \phi\right)$. The jump conditions are therefore considered in a vertical plane under pure potential-flow conditions with $v=0$ and thus $\bar{u}=\overline{\partial_{x} \phi}$. The horizontal momentum equation arising from (21b) and the continuity equation read

$h \partial_{x}\left(\left(\partial_{t} \phi\right)_{s}+\frac{1}{2}|\nabla \phi|_{s}^{2}+g(h+b)\right)=0$ and $\partial_{t} h+\partial_{x}(h \bar{u})=0$,

or after some reworking:

$\partial_{t}(h \bar{u})+\partial_{x}\left(h \bar{u}^{2}+\frac{1}{2} g h^{2}\right)+g h \partial_{x} b+h\left(\partial_{x}\left(\partial_{t} \phi\right)_{s}-\partial_{t} \bar{u}\right)+\frac{1}{2} h \partial_{x}\left(|\nabla \phi|_{s}^{2}-\bar{u}^{2}\right)=0 \quad$ and $\quad \partial_{t} h+\partial_{x}(h \bar{u})=0$

with $\nabla=\left(\partial_{x}, \partial_{z}\right)^{T}$ in a vertical plane. Formulation of the weak formulation directly from (53), or simplification to one dimension from (51), yields

$$
\begin{aligned}
& \left\{\begin{array}{l}
{[h(S-\bar{u})]=0} \\
-S[h \bar{u}]+\left[h \bar{u}^{2}+g h^{2} / 2\right]+\frac{[\{h\}]\left[g\{h\} b+h\left(\left(\partial_{t} \phi\right)_{s}+S \bar{u}\right)+\frac{1}{2} h\left(|\nabla \phi|_{s}^{2}-\bar{u}^{2}\right)\right]}{-[h] \int_{0}^{1}\left(\left(\partial_{t} \widetilde{\phi)_{s}+} S \bar{u}\right)\left(\tau ; U^{-}, U^{+}\right)+\frac{1}{2}\left(\mid\left(\nabla \phi \widehat{)\left._{s}\right|^{2}-}|\bar{u}|^{2}\right)\left(\tau ; U^{-}, U^{+}\right) \mathrm{d} \tau=0\right.\right.}
\end{array}\right.
\end{aligned}
$$

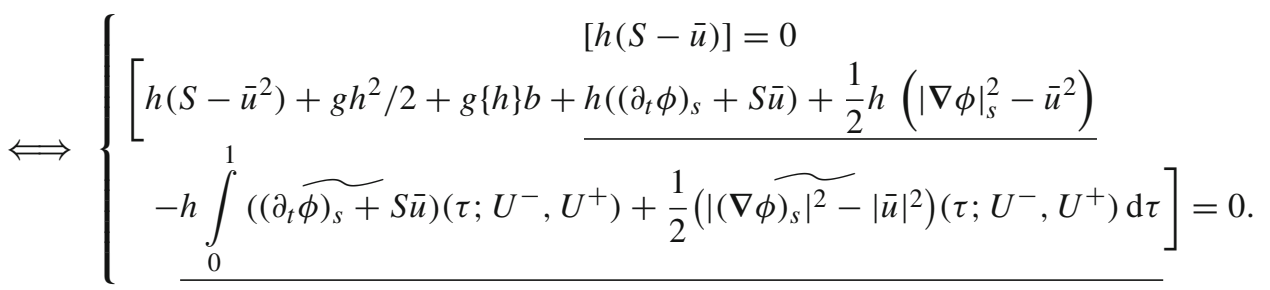

Consider the simple case with constant depths $h_{ \pm}, h_{-}>h_{+}$, and depth-averaged velocities $\bar{u}_{ \pm}$on either side of a steady jump, localized at $x=0$ and over a flat bottom $b=0$. The underlined terms then cancel and the shallow-water bore relations remain. Hence, $\phi_{ \pm}=\bar{u}_{ \pm} x$ plus a dependence on $z$ which is zero as follows from Laplace's equation and the bottom boundary condition $w=\partial_{z} \phi_{ \pm}=0$. The conditions on $\phi$ at $x=0$ are therefore Dirichlet as $\phi$ is continuous below the vertical jump face: $0<z<h_{+}$; and, Neumann on the vertical jump face: $\left(\partial_{x} \phi\right)_{-}=\bar{u}_{-}-S=\bar{u}_{-}$as the jump is assumed to be steady.

This simple example shows what the extra boundary conditions become at the vertical face. In general, the condition is that the horizontal velocity normal to the jump equals the "upstream" depth-averaged velocity in the frame of reference of the jump: $\overline{\boldsymbol{u}}_{-} \cdot \hat{\boldsymbol{n}}-S_{n}=(\hat{\boldsymbol{n}}, 0)^{T} \cdot(\nabla \phi+\mathbf{v})_{-}$for the case with $h_{-}>h_{+}$. Finally, we re-emphasize that these results are somewhat heuristic because we applied a theory of weak solutions for hyperbolic-equations with non-conservative products to coupled hyperbolic-elliptic equations.

\subsection{Linear waves}

Linearization of (36) around a state of rest and over a flat bottom yields $\mathbf{v}=0$. Hence, the dispersion relation for the classical water-wave equations holds again for our extended water-wave equations. For linear flows over non-uniform topography the dispersion relation will be an extension of both the water-wave results under potential flow and the shallow-water equations. 


\section{Concluding remarks}

The aims laid out in the introduction have been reached nearly completely. We have derived a reduced model with accurate surface water-wave dispersion and vertical vorticity. It is derived systematically via a simplification of the variational principle of the three-dimensional incompressible Euler equations with free surface dynamics. We noted that associated conservation laws follow from the symmetries of our new variational principle. Mass, momentum, and energy conservation and material conservation of depth-averaged potential vorticity are thus guaranteed. The model is reduced, or simplified, because it contains only the depth-averaged vertical vorticity combined with the full three-dimensional velocity potential. On the one hand, our new model reduces to the classical depth-averaged shallow-water equations in the shallow-water limit, and, on the other hand, it reduces to the classical potential-flow water-wave equations in the irrotational limit. Breaking waves can be included in our new model by extension of the classical bore relations in the usual depth-averaged shallow-water equations. The multivalued free surface of an overturning wave is therefore limited locally to a vertical face. The advanced jump relations are based on the continuity equation and an analysis of the depth-averaged part of the momentum equations. It is an heuristic extension of the theory of shocks in hyperbolic equations with nonconservative products. Nonetheless, these extended shock relations clearly reduce to the classical bore relations for depth-averaged shallow-water equations in the shallow-water limit. Further analysis needs to sort out what the associated loss of energy and possibly some momentum is. For flow over a flat bottom, linear waves in our new model coincide with those for the irrotational water-wave equations.

It is important to stress that the preservation of the geometric structure in an Eulerian model is non-trivial. Our usage of the combination potential flow plus the depth-averaged vortical velocity component is special because it allows the preservation of the relabelling symmetry with its associated potential vorticity conservation material or integral laws [15,24]. The following remains an outstanding research question: Can we preserve the geometric (Hamiltonian) structure in Eulerian variables for higher-order discretizations of the vorticity vector as function of vertical coordinate $z$ ? It goes without saying that conservation of energy and perhaps momentum is locally broken through a hydraulic jump or bore.

Two issues we aimed at have not yet been resolved. The first unresolved issue is that the new model is still quite complex as it contains the full three-dimensional potential-flow component. The derivation of our new model with this potential-flow dependence was necessary to pinpoint the coupling between the potential-flow part and the vortical part. Further approximations of the velocity potential (e.g., by a quadratic polynomial in the vertical $z$-direction) and subsequent substitution thereof in the variational principle immediately leads to a Boussinesq-type model, with high-order accurate dispersion and vertical vorticity. Any such substitution and evaluation within the variational principle is permissible and straightforward; and, it guarantees preservation of the conservative structure. In essence, it constitutes a discretization in the vertical direction only; the three-dimensional Laplace equation emerging in the water-wave part of our model is then discretized in the vertical and replaced by an elliptical equation in the horizontal coordinates with non-constant depth-dependent coefficients. Such a simplification to Boussinesq-type models follows immediately from work of [13] which, however, only applies consistently to potential flows. Furthermore, it may be easier to recombine the potential and vortical part in a formulation with a depth-averaged velocity after such an additional Boussinesq-type simplification has been made. The second unresolved issue concerns the analysis of generation of potential vorticity for bores in which energy is dissipated in a non-uniform manner along the breaking-wave crest. Such an analysis is available for the depth-averaged shallow-water equations [6,7], and should be made for our new model.

Future work will also involve discontinuous Galerkin finite-element discretizations (DGFEM) of our new model, or Boussinesq versions thereof. It will combine our research on shallow-water ocean models [28], on (numerical) generation of potential vorticity by non-uniform bores [11,12] and on flooding and drying [29]. Our DGFEM discretization based on a Luke's variational principle [21,30] will be used herein as a building block. In addition, we aim to explore exact solutions, inspired by [31]. Finally, the Lagrangian (14) for the three-dimensional incompressible Euler flow and the one for our new wave model (30) are singular. In both cases the constraint $D-1=0$ (of density being constant) has as consistency constraint the corresponding incompressibility condition. Consistency 
of the latter then leads to an elliptic equation for the pressure. Dirac's theory of constraint Lagrangians [32] would explicitly establish a formulation of the dynamics on the constraint manifold. In [33], an example is given for shallow-water flow constrained to constant depth and incompressibility, which may be extended to the free-surface case both for the three-dimensional parent model and our new water-wave model. Bokhove [34] also offers an alternative view, with similar results as Dirac's theory, based on slaved Hamiltonian dynamics. The latter could be more appealing given the free-surface boundary conditions, compared with the approach followed in [33].

Acknowledgments The photograph "sun-on-bore", as the late Professor Howell D. Peregrine (DHP) called it, in Fig. 3a featured most prominently on the poster "maths makes waves" (Fig. 4) in the campaign "World Mathematical Year 2000, Posters in the London Underground". Howell Peregrine was always keen that his photographs were used for good, whether it be in the sciences or (fine) arts. O.B. was a postdoctoral research associate with DHP (and Professor Andrew Woods) in 1998 and 1999 at the School of Mathematics in Bristol, U.K. Thereafter, our scientific, geological and walking tours continued, till March 2007. We thank Sander Rhebergen and Shavarsh Nurijanyan for proofreading several sections of the manuscript. Finally, it is a pleasure to thank the organizers of two workshops, Geometric and Stochastic Methods in Geophysical Fluid Dynamics in Bremen 2008 (a.o., Marcel Oliver) and Numerical Modelling of Complex Dynamical System at the Lorentz Center in 2008 (a.o., Jason Frank), for providing the seeding opportunities of the research presented.

Open Access This article is distributed under the terms of the Creative Commons Attribution Noncommercial License which permits any noncommercial use, distribution, and reproduction in any medium, provided the original author(s) and source are credited.

\section{Appendix: Variational preliminaries}

First consider the connection (5), in the main text, between the variation of the velocity $\delta \boldsymbol{u}$ and the displacement $\mathbf{w}$; it arises from (56), to wit

$\partial_{t} \chi=\boldsymbol{u} \circ \chi \equiv \boldsymbol{u}(\chi, t)$ and $\delta \chi=\mathbf{w} \circ \chi \equiv \mathbf{w}(\chi, t)$.

Equating the variation of the first and the time derivative of the second equation in (56) yields

$\delta\left(\boldsymbol{u}_{i} \circ \chi\right)=\partial_{t}\left(\mathbf{w}_{i} \circ \chi\right) \Longleftrightarrow\left(\delta \boldsymbol{u}_{i}\right) \circ \chi+\delta \chi_{j} \cdot \nabla_{j} \boldsymbol{u}_{i} \circ \chi=\left(\partial_{t} \mathbf{w}\right)_{i} \circ \chi+\partial_{t} \boldsymbol{\chi}_{j} \cdot \nabla_{j} \mathbf{w}_{i} \circ \chi$,

where we superfluously added indices for clarity. Hence, by rearranging (57) one finds that (5) follows:

$\left(\delta \boldsymbol{u}_{i}\right) \circ \chi=\left(\partial_{t} \mathbf{w}\right)_{i} \circ \chi+\partial_{t} \chi_{j} \cdot \nabla_{j} \mathbf{w}_{i} \circ \chi-\delta \chi_{j} \cdot \nabla_{j} \boldsymbol{u}_{i} \circ \chi \Longrightarrow \delta \boldsymbol{u}_{i}=\partial_{t} \mathbf{w}_{i}+\boldsymbol{u}_{j} \cdot \nabla_{j} \mathbf{w}_{i}-\mathbf{w}_{j} \cdot \nabla_{j} \boldsymbol{u}_{i}$.

Second, consider the Jacobian $\boldsymbol{J}$ between Eulerian $\boldsymbol{x}=\left(x_{1}, x_{2}, x_{3}\right)^{T}$ and Lagrangian coordinates $\boldsymbol{l}=$ $\left(l_{1}, l_{2}, l_{3}\right)^{T}$, defined as:

$\boldsymbol{J}=\left(\begin{array}{lll}\partial_{x} l_{1} & \partial_{y} l_{1} & \partial_{z} l_{1} \\ \partial_{x} l_{2} & \partial_{y} l_{2} & \partial_{z} l_{2} \\ \partial_{x} l_{3} & \partial_{y} l_{3} & \partial_{z} l_{3}\end{array}\right)$.

The determinant of this Jacobian is

$\epsilon_{i j k}\left(\partial_{x_{i}} l_{1}\right)\left(\partial_{x_{j}} l_{2}\right)\left(\partial_{x_{k}} l_{3}\right)$

with indices $i, j, k=1,2,3$ and permutation symbol $\epsilon_{i j k}$ such that $\epsilon_{123}=1$ and likewise for cyclic shifts, and $\epsilon_{321}=-1$ and likewise for cyclic shifts, and $\epsilon_{i j k}=0$ when at least two indices coincide. Hence, the variation of the determinant yields:

$$
\begin{aligned}
\delta D & =\partial_{x_{i}}\left(\epsilon_{i j k}\left(\partial_{x_{j}} l_{2}\right)\left(\partial_{x_{k}} l_{3}\right) \delta l_{1}+\epsilon_{j i k}\left(\partial_{x_{j}} l_{1}\right)\left(\partial_{x_{k}} l_{3}\right) \delta l_{2}+\epsilon_{k j i}\left(\partial_{x_{k}} l_{1}\right)\left(\partial_{x_{j}} l_{2}\right) \delta l_{3}\right) \\
& \equiv-\partial_{x_{i}}\left(\mathrm{w}_{i} D\right) .
\end{aligned}
$$

The last definition of $\mathbf{w}$ defines the link between the variation of $\boldsymbol{\chi}$ and $\delta \boldsymbol{l}$, as follows

$\mathbf{w}=-\boldsymbol{J}^{-1} \delta \boldsymbol{l}$. 
Likewise, one can show that $\boldsymbol{u}=-\boldsymbol{J}^{-1} \partial_{t} \boldsymbol{l}$. Alternatively, this link can be established in an integral way as

$$
\begin{aligned}
\delta D(x, y, z, t)= & \delta \int_{\Omega^{\prime}} D\left(x^{\prime}, y^{\prime}, z^{\prime}, t\right) \delta\left(\boldsymbol{x}-\boldsymbol{x}^{\prime}\right) \mathrm{d} x^{\prime} \mathrm{d} y^{\prime} \mathrm{d} z^{\prime} \\
= & -\int_{\Omega^{\prime}} \nabla \delta\left(\boldsymbol{x}-\chi\left(l_{1}, l_{2}, l_{3}, t\right)\right) \cdot \delta \chi\left(l_{1}, l_{2}, l_{3}, t\right) \mathrm{d} l_{1} \mathrm{~d} l_{2} \mathrm{~d} l_{3} \\
= & \int_{\Omega^{\prime}} \nabla^{\prime} \delta\left(\boldsymbol{x}-\boldsymbol{x}^{\prime}\right) \cdot \mathbf{w}\left(x^{\prime}, y^{\prime}, z^{\prime}, t\right) D\left(x^{\prime}, y^{\prime}, z^{\prime}, t\right) \mathrm{d} x^{\prime} \mathrm{d} y^{\prime} \mathrm{d} z^{\prime} \\
= & -\int_{\Omega^{\prime}} \delta\left(\boldsymbol{x}-\boldsymbol{x}^{\prime}\right) \nabla^{\prime} \cdot\left(\mathbf{w}\left(x^{\prime}, y^{\prime}, z^{\prime}, t\right) D\left(x^{\prime}, y^{\prime}, z^{\prime}, t\right)\right) \mathrm{d} x^{\prime} \mathrm{d} y^{\prime} \mathrm{d} z^{\prime} \\
& +\int_{\partial \Omega^{\prime}} \delta\left(\boldsymbol{x}-\boldsymbol{x}^{\prime}\right) D\left(x^{\prime}, y^{\prime}, z^{\prime}, t\right) \mathbf{w}\left(x^{\prime}, y^{\prime}, z^{\prime}, t\right) \cdot \hat{\boldsymbol{n}} \mathrm{d} s^{\prime} \\
= & -\nabla \cdot(\mathbf{w} D),
\end{aligned}
$$

since $\delta\left(\boldsymbol{x}-\boldsymbol{x}^{\prime}\right)=0$ on $\partial \Omega$ in the interior of the fluid, and with the primes indicating evaluation at $\boldsymbol{x}^{\prime}=\left(x^{\prime}, y^{\prime}, z^{\prime}\right)^{T}$. With some care of the treatment at the free surface, a similar derivation and relation hold at the free surface. It is then important to note and use that $\delta\left(D_{s}\right)=(\delta D)_{s}+\left(\partial_{z} D\right)_{s} \delta h$. Thus, we have proved expression (7) in two ways.

Finally, consider a general representation $F=F(x, y, z, t)=0$ of the free surface. The variation of the position of the fluid parcel should be zero at the free surface, just like the time variation on the free surface following the fluid parcel remains zero. Hence,

$0=\delta F=(\delta F)_{s}+(\mathbf{w} \cdot \nabla F)_{s}$.

For the restrictive particularization $F=z-(h+b)$ used so far, we thus find (9), i.e.:

$0=-\delta h-\mathbf{w}_{S} \cdot \nabla_{H}(h+b)+\mathbf{w}_{S}$.

Likewise, one finds the kinematic condition (8).

Integration of (64) over depth, usage of (66), and free-slip boundary conditions at fixed walls yields a version

$\delta(h \bar{D})+\nabla_{H} \cdot(h \overline{D \mathbf{w}})=0$

with a depth-integrated density and horizontal-label variations

$h \bar{D}=\int_{b}^{b+h} D \mathrm{~d} z$ and $h \overline{D \mathbf{w}}=\int_{b}^{b+h} D\left(\mathrm{w}_{1}, \mathrm{w}_{2}\right)^{T} \mathrm{~d} z$.

Likewise, a depth-averaged continuity equation can be derived. For incompressible flows with $D=1$ these depthaveraged equations yield (10) in the main text.

The above calculations reveal why it is useful to use $\mathbf{w}$ instead of $\delta \boldsymbol{l}$ : it is a concise Eulerian representation of $\delta \chi$ significantly economizing notations and interpretations.

\section{References}

1. Salmon R (1998) Geophysical fluid dynamics. Oxford University Press, Oxford

2. Stoker JJ (1957) Water waves, Chap 10. Interscience Publications, Wiley, New York

3. Peregrine DH (1983) Breaking waves on beaches. Annu Rev Fluid Mech 15:149-178

4. Peregrine DH (1967) Long waves on a beach. J Fluid Mech 27:815-827

5. Agnon Y, Madsen PA, Schäffer HA (1999) A new approach to high-order Boussinesq models. J Fluid Mech 399:319-333

6. Pratt L (1983) On inertial flow over topography, Part I: Semigeostrophic adjustment to an obstacle. J Fluid Mech 131:195-218 
7. Peregrine DH (1998) Surf zone currents. Theor Comput Fluid Dyn 10:295-310

8. Peregrine DH (1999) Large-scale vorticity generation by breakers in shallow and deep water. Eur J Mech B Fluids 18:403-408

9. Peregrine DH, Bokhove O (1998) Vorticity and surf zone currents. In: Edge BL (ed) 26th international conference on coastal engineering, Reston ASCE, Copenhagen, pp 745-758. ISBN: 0-7844-0411-9

10. Bühler O (2000) On the vorticity transport due to dissipating or breaking waves in shallow-water flow. J Fluid Mech 407:235-263

11. Ambati VR, Bokhove O (2007) Space-time discontinuous Galerkin discretization of rotating shallow water equations. J Comput Phys 225:1233-1261

12. Tassi P, Bokhove O, Vionnet C (2007) Space discontinuous Galerkin method for shallow-water flows-kinetic and HLLC flux, and potential vorticity-generation. Adv Water Resour 30:998-1015

13. Klopman G, Dingemans M, van Groesen B (2007) Propagation of wave groups over bathymetry using a variational Boussinesq model. In: Proceedings of the 22nd international workshop on water waves and floating bodies, conference paper. http://eprints. eemcs.utwente.nl/

14. Miles J, Salmon R (1985) Weakly dispersive nonlinear gravity waves. J Fluid Mech 157:519-531

15. Salmon R (1988) Hamiltonian fluid mechanics. Annu Rev Fluid Mech 20:225-256

16. Camassa R, Holm DD, Levermore CD (1997) Long-time shallow-water equations with a varying bottom. J Fluid Mech 349:173-189

17. Luke JC (1967) A variational principle for a fluid with a free surface. J Fluid Mech 27:395-397

18. Miles J (1985) Surface waves in basins of variable depth. J Fluid Mech 83:153-158

19. Malandain JJ (1988) La Seine au Temps du Mascaret. (The Seine River at the Time of the Mascaret.) Le Chasse-Maré 34:30-45 (in French)

20. Holm DD, Marsden JE, Ratiu TS (1998) The Euler-Poincaré equations and semidirect products with applications to continuum theories. Adv Math 137:1-81

21. Ambati VR, van der Vegt JJW, Bokhove O (2008) Variational space-time (dis)continuous Galerkin method for free surface waves (submitted). http://eprints.eemcs.utwente.nl/

22. Green AE, Laws N, Naghdi PM (1974) On the theory of water waves. Proc R Soc Lond A 388:43-55

23. Percival JR, Cotter CJ, Holm DD (2008) A Euler-Poincaré framework for the multilayer Green-Nagdhi equations. J Phys A Math Theor 41:344018

24. Cotter CJ, Holm DD, Hydon PE (2007) Multisymplectic formulation of fluid dynamics using the inverse map. Proc Roy Soc A 463:2671-2687

25. Dal Maso G, LeFloch PG, Murat F (1995) Definition and weak stability of nonconservative products. J Math Pures Appl 4:483-548

26. Parés C (2006) Numerical methods for nonconservative hyperbolic systems: a theoretical framework. SIAM J Numer Anal 44:300321

27. Rhebergen S, Bokhove O, Vegt JJW (2008) Discontinuous Galerkin finite element methods for hyperbolic nonconservative partial differential equations. J Comput Phys 227:1887-1922

28. Cotter CJ, Ham DA, Pain CC (2009) A mixed discontinuous/continuous finite element pair for shallow-water ocean modelling. Ocean Model 26:86-90

29. Bokhove O (2005) Flooding and drying in finite-element Galerkin discretizations of shallow-water equations. Part I: One dimension. J Sci Comput 22:47-82

30. Ambati VR (2008) Forecasting water waves and currents: a space-time approach. Ph.D. dissertation, University of Twente. http:// eprints.eemcs.utwente.n1/ pp. 151

31. Constantin A (2007) Edge waves along a sloping beach. J Phys A Math Gen 34:9723-9731

32. Dirac PAM (1950) Generalized Hamiltonian dynamics. Can J Math 2:129-148

33. Salmon R (1988b) Semigeostrophic theory as a Dirac-bracket projection. J Fluid Mech 196:345-358

34. Bokhove $\mathrm{O}$ (2002) Balanced models in geophysical fluid dynamics: Hamiltonian formulation, constraints and formal stability, Chap 1, 63 pp. In: Norbury J, Roulstone I (eds) Large-scale atmosphere-ocean dynamics 2, geometric methods and models. Cambridge University Press, 364 pp 